AperTO - Archivio Istituzionale Open Access dell'Università di Torino

\title{
PARP Inhibitors in Ovarian Cancer
}

\section{This is a pre print version of the following article:}

Original Citation:

\section{Availability:}

This version is available http://hdl.handle.net/2318/1742207

since 2020-06-23T22:11:53Z

Published version:

DOI:10.2174/1574892813666180305165256

Terms of use:

Open Access

Anyone can freely access the full text of works made available as "Open Access". Works made available under a Creative Commons license can be used according to the terms and conditions of said license. Use of all other works requires consent of the right holder (author or publisher) if not exempted from copyright protection by the applicable law. 


\section{PARP inhibitors in ovarian cancer}

Mittica Gloria $\mathrm{MD}^{1,2}$, Ghisoni Eleonora $\mathrm{MD}^{1,2}$, Giannone Gaia $\mathrm{MD}^{1,2}$, Genta Sofia $\mathrm{MD}^{1,2}$, Aglietta Massimo $\mathrm{MD}^{1,2}$, Sapino Anna $\mathrm{MD}^{2,3}$, Valabrega Giorgio $\mathrm{MD}^{1,2^{*}}$

${ }^{1}$ Department of Oncology, University of Torino, Italy

${ }^{2}$ Candiolo Cancer Institute-FPO-IRCCS, Candiolo, Torino, Italy

${ }^{3}$ Department of Medical Sciences, University of Torino, Italy

Email addresses: gloria.mittica@ircc.it; $\quad$ eleonora.ghisoni@ircc.it; gaia.giannone@ircc.it; sofia.genta@ircc.it; massimo.aglietta@ircc.it; anna.sapino@ircc.it; giorgio.valabrega@ircc.it

Running title: Role of PARP inhibitors for the treatment of ovarian cancer: current status and future perspectives.

* Corresponding author: Giorgio Valabrega

giorgio.valabrega@ircc.it

$\mathrm{Ph}+\underline{+390119933253}$

FAX +390119933275

Department of Oncology, Candiolo Cancer Institute-FPO-IRCCS

Strada provinciale $142 \mathrm{~km} \mathrm{3.95,} 10060$ Candiolo, Torino, Italy 


\begin{abstract}
Background: Treatment of Epithelial Ovarian Cancer (EOC), historically based on surgery and platinum doublet chemotherapy, is associated with high risk of relapse and poor prognosis for recurrent disease. In this landscape, the innovative treatment with PARP inhibitors (PARPis) demonstrated an outstanding activity in EOC, and is currently changing clinical practice in BRCA mutant patients.
\end{abstract}

Objectives: To highlight the mechanism of action, pharmacokinetics, clinical activity, indications and current strategies of development of Olaparib, Niraparib, Rucaparib, Talazoparib and Veliparib, the 5 most relevant PARPis.

Methods: We performed a review on Pubmed using 'ovarian cancer' and the name of each PARPi (PARP inhibitor) discussed in the review as Medical Subject Headings (MeSH) keywords. The same search was performed on "clinicaltrial.gov" to identify ongoing clinical trials and on "google.com/patents" and "uspto.gov" for recent patents exploring PARPIs in ovarian cancer.

Results: Olaparib, Niraparib and Rucaparib are already approved for treatment of recurrent EOC and their indications are partially overlapping. Talazoparib and Veliparib are promising PARPis, but currently under investigation in early phase trials. Several studies are evaluating PARPis in monotherapy or in associations, in a wide range of settings (i.e. first line, neoadjuvant, platinumsensitive and resistant disease).

Conclusion: PARPis are valuable options in patients with recurrent ovarian cancer with promising activity in different stages of this disease. Further studies are required to better define optimal clinical settings, predictors of response beyond BRCA mutations and strategies to overcome secondary resistance of PARPis therapy in EOC.

Keywords: ovarian cancer, PARP inhibitors, olaparib, niraparib, rucaparib, veliparib, talazoparib, recent patents. 


\section{INTRODUCTION}

Epithelial ovarian cancer (EOC) is the most lethal gynaecological malignancy with 22440 estimated new cases and 14080 deaths in the United States in 2017 [1]. It is frequently diagnosed at advanced stage and standard therapeutic strategy consists of optimal debulking surgery (absence of residual disease) and platinum-based chemotherapy [2]. However, despite optimal surgery, about $70 \%$ of patients experience relapse [3]. Probability of response and prognosis of relapsed patients is related to progression/relapse free interval from last platinum therapy (Platinum free interval, PFI). Patients with platinum-refractory (PFI within 4 weeks) and platinum resistant (PFI within 6 months) disease would receive a single-agent chemotherapy (above all Pegylated Liposomal Doxorubicin-PLD, Taxanes, Gemcitabine, Etoposide or Topotecan) with low response rate and poor prognosis. On the other hand, patients that are platinum partially-sensitive (PFI between 6 and 12 months) and platinum sensitive (PFI longer than 12 months) could receive a platinum based therapy with a better prognosis and longer survival [2]. In this landscape, characterized by few therapeutic options and a narrow range of drugs that improve survival, inhibitors of poly (ADP-ribose) polymerases (PARPis) emerged as an exciting option [4]. Olaparib, Rucaparib, Niraparib, Veliparib and Talazoparib are the most studied PARPis and the first three have already been approved in different setting of relapsed EOC, determining a radical change in current practice.

\subsection{DNA repair and Homologous Recombination Deficiency}

DNA damage is a frequent event during cell life; it can be spontaneous (e.g. replication error) or caused by cell metabolism or by environmental agents[5]. It can result in single strand DNA breaks (SSBs) or double strand DNA breaks (DSBs) that cause loss of genome integrity and cell death, if not correctly repaired [6]. There are six main DNA repair pathways [7] [8]. Mismatch Repair (MMR), Nucleotide Excision Repair (NER), Base Excision Repair (BER), Trans-lesional Synthesis, Non Homologous end joining (NHEJ), and Homologous recombination (HR) [9]. The last two pathways are responsible for repairing DSBs; HR is a high fidelity repair mechanism, active during Phase G2-S of cell cycle[10, 11], whereas NHEJ is faster but error prone [12] [13]. If HR pathway is altered, leading to Homologous Recombination Deficiency (HRD), cells rely above all on NHEJ [14], with a less preserved genomic integrity and a higher risk to develop cancers [12] [13]. The first HR proteins that have been studied were Breast Related Cancer Antigen (BRCA) 1[15] [16] and 2 [17], whose germline mutations and, consequently, loss of function were correlated with an increased risk of breast cancer (ranging from 57\% in BRCA1 mutation to $45 \%$ in BRCA2 mutation) and ovarian cancer (ranging from $11 \%$ in BRCA 1 mutation to $40 \%$ in BRCA2 mutation) [18] [19]. 
BRCA mutated OC display distinctive biological and clinical features[20], being usually high grade cancers (G2-G3), with serous or undifferentiated histology [21]. Moreover, they are frequently diagnosed at an advanced stage [22] and in young women but BRCA mutated patients have a better prognosis in comparison with BRCA wild type (BRCA WT) EOC. [21] [23] [24]. Until recent approval of the new drugs, PARPis, patients with gBRCAm EOC had the same treatment of BRCA wild type EOC [25] [26] [27]. Recently TCGA demonstrated that almost half of high grade serous ovarian cancer (HGSOC), the most frequent EOC histology, exhibits HRD [28]. Germline BRCA1 and 2 [22] [29] account for $22.6 \%$ of mutations in HGSOC, usually accompanied by loss of heterozygosis $(\mathrm{LOH})$, while somatic mutations are present in 6-7\% [30] and BRCA1 hypermethylation is found in about $10 \%$ of HGSOC [28] Other altered genes in HRD pattern are EMSY (8\%), PTEN (Phosphatase and Tensin Homolog) (7\%), RAD 51C (3\%), ATM/ATR (Ataxia-telangiectasia mutated and ATM- and RAD3-related) (2\%), Fanconi Anemia genes (5\%) and RAD $50(<1 \%)$ [28]. Sporadic EOCs that exhibit HRD without BRCA 1 or BRCA 2 mutations have similar histology, biology and clinical features of BRCA mutated tumours (so called BRCAness phenotype [31] [32] [33]). Unfortunately, nowadays a standardized test that can optimally identify HRD EOCs does not exist, even if several strategies were attempted. Among them some have tried to identify directly the key mutations in HR pathway with gene expression profiles of DNA repair proteins using "targeted capture and massively parallel sequencing" (e.i. BROCA profile) [34] [35] [36]. Other tests used indirect 'scars' [37], such as mutational signatures [38] or structural variations of the genome, identified as genome variation larger than $100 \mathrm{~kb}$, that result from DSB in cells with HRD [37]. The most important "scars" used in HRD tests are:

- Numerical chromosomal instability (CIN), an amplification or deletion of large fragments of chromosomes, usually interesting a whole chromosome arm [39] [40] [41];

- Somatic rearrangement pathways related to specific DNA-repair deficiency, for example tandem duplications (TDs) [42] ;

- Loss of Heterozygosis ( $\mathrm{LOH}$ ) of multiple megabasis fragments [43], used as marker of HRD in several trials as discussed below [26] [44].

- A combination of three variables: intermediate size $\mathrm{LOH}(>15 \mathrm{Mb})$ added to allelic imbalance that involve the telomerase (TAI) and number of chromosomal breaks longer than $10 \mathrm{Mb}[45][27]$.

\subsection{PARP family and PARP inhibitors Mechanism of action}


In the complex landscape of DNA repair mechanism, PARP family plays a central role [46]. PARPs are a group of enzymes that transfer polyADP-ribose from NAD+ to some target proteins in a post transcriptional process known as PARylation [47]. Among them, PARP-1, 2 and 3 are the most extensively studied. PARP-1, that accounts for up to $90 \%$ of the entire PARP activity, recruits DNA repair complex in BER [48], detects disruptions in replication forks [49], PARylates essential proteins involved in HR [50] (e.i. BRCA1 recruitment to sites of DNA damage [51]) and acts as a negative regulator in NHEJ [52] [53], with the cooperation of PARP-2 and PARP-3 and plays a central role in microhomology-mediated end joining repair, an error-prone pathway involved in DSBs repair [52] [53]. Beyond DNA repair PARPs are also involved in transcriptional regulation, mitosis, cell death, telomere length and intracellular metabolism [54].

PARPis are generally benzamide or purine based analogues of nicotinamide that compete with NAD+ in the catalytic domain of PARPs [55] [56]. Their activity is historically based on the concept of "synthetic lethality", first introduced in 2005, according to which two genetic lesions, not lethal when individually present, become lethal if both occur in the same cell. For this reason cells that are HR deficient are more sensitive to PARP activity inhibition [50] [57] [58]. PARP inhibition reduces both HR and BER activity, thus SSBs remaining unrepaired [59], leading to a high number of DSBs that are not repaired in HRD cells during DNA duplication [59]. PARPis prevent also the inhibition of NHEJ, with a high load of mutations and cell apoptosis [60]. Figure 1 resumes the principal PARPis mechanisms of action above described.

All PARPis [54] [61] developed in EOC are PARP-1 and PARP-2 inhibitors, while Olaparib and Rucaparib inhibit also PARP-3 and Rucaparib inhibits also tankyrase-1, another member of PARP family[62] [63].

Recent studies show that PARP inhibition is more cytotoxic that genetic depletion of PARPs, finding that PARPis "stabilized" PARP-1 and PARP-2 DNA complexes. This activity, known as PARP trapping, prevents DNA repair, leading to cell death [62] [63]. Murai et al demonstrated that PARP trapping is strongly related to PARPis cytotoxic activity [62] [63] [64]. Despite of similar PARP catalytic inhibitory activities, Talazoparib shows the greatest in vitro cytotoxicity and PARPtrapping [63], followed by Niraparib. Olaparib and Rucaparib show a medium citotoxicty and PARP-trapping, whereas Veliparib is the weakest one [4] [62]. These differences are probably due to allosteric differences in NAD+ binding site; Niraparib and Olaparib are bulky inhibitors compared to Veliparib, while Talazoparib has a rigid structure if compared to the "flexible" Olaparib and Rucaparib [62] [63]. 
The aim of this review is to describe the rationale beyond development of PARPis, their mechanism of action and the most important clinical trial that led to their approval in clinical practice. Moreover, we report an overview and future perspectives of these new molecules summarizing the ongoing clinical trial with PARPis. To do this we operated a search on clinicaltrials.gov with "ovarian cancer" and the single name of each compound (Olaparib, Niraparib, Rucaparib, Veliparib and Talazoparib) as keywords.

\section{OLAPARIB}

\subsection{Description}

Olaparib (@Lynparza, Astra Zeneca), known as AZD2281, is an inhibitor of the mammalian polyadenosine 5'-diphosphoribose polymerase (PARP) enzymes 1, 2 and 3 (PARP 1, PARP2 and PARP 3 respectively). Its chemical name is 4-[(3-\{[4-(cyclopropylcarbonyl) piperazin-1yl]carbonyl -4-fluorophenyl)methyl]phthalazin-1(2H)] one and the chemical structure is showed in Figure 2. Olaparib molecular formula is C24H23FN43 and has a molecular mass of $434.46 \mathrm{amu}$. Lynparza has a low, $\mathrm{pH}$-indipendent, solubility, with a value of nearly $0.1 \mathrm{mg} / \mathrm{ml}$ in the normal $\mathrm{pH}$ range.

The molecule is disposable for oral use as capsules since 2014, each one containing $50 \mathrm{mg}$ of Olaparib associated with some excipients [65]. Since August 2017, after the publication of SOLO-2 study results (see below for the details), also a tablet formulation is available in two different dosages, containing respectively $100 \mathrm{mg}$ and $150 \mathrm{mg}$ of Olaparib

\subsection{Pharmacokinetics}

Comparing tablet and capsule formulation the bioavailability is higher for the tablets, with a steady state exposure (AUC) 77\% higher after $300 \mathrm{mg}$ tablet twice daily in comparison after 400mg capsule twice daily. After a single dose of $300 \mathrm{mg}$ tablet the geometric mean AUC is $42 \mu \mathrm{g}^{*} \mathrm{~h} / \mathrm{ml}$, while $\mathrm{C}_{\max }$ is $5.8 \mu \mathrm{g} / \mathrm{ml}$, while the steady state data are $49 \mu \mathrm{g}^{*} \mathrm{~h} / \mathrm{ml}$ and $7.7 \mu \mathrm{g} / \mathrm{ml}$ respectively. In the pharmacodynamics studies no clinically relevant alterations of cardiac repolarization neither modification in QT interval were reported[66].

\section{Absorption}

After oral intake the median peak plasma concentration is established after 1.5 hours. The coadministration of high fat meals prolonged that time, with a delay of 2.5 hours in the absorption, but 
no significant alteration in the amount of absorption was reported, except an increased mean AUC of nearby $8 \%$.

\section{Distribution}

The mean $( \pm \mathrm{SD})$ apparent volume of distribution is $158 \pm 136 \mathrm{~L}$ after a single intake of $300 \mathrm{mg}$ of Olaparib, with an in vitro protein binding nearly $82 \%$.

\section{Metabolism}

The two enzymes mostly involved in Olaparib metabolism are CYP3A4 and CYP3A5. For that reason, concomitant use of strong or moderate CYP3A inhibitors should be avoided. Oxidation reactions are fundamental in the metabolism and the great part of metabolites are produced with glucuronide or sulfate conjugation.

\section{Excretion}

After a single $300 \mathrm{mg}$ dose the mean $( \pm \mathrm{SD}$ ) plasma half-life is $14.9 \pm 8.2$ hours, with an apparent plasma clearance of $7.4 \pm 3.9 \mathrm{~L} / \mathrm{h}$. In an experiment with ${ }^{14} \mathrm{C}$-Olaparib, $86 \%$ of radioactivity is redeemed in 7 days, $44 \%$ through urine and $42 \%$ through feces, with the most of compound excreted as metabolites [67].

\subsection{First evidence of clinical activity}

Olaparib was the first small-molecule oral PARP inhibitor developed in ovarian cancer. Back in 2009 a phase I trial by Fong and colleagues reported a low side effect profile (fatigue, nausea and anaemia in $30 \%, 32 \%$ and $5 \%$ of patients respectively) associated with a clinical benefit of $63 \%$ in BRCA $1 / 2$ mutated population (19 patients) [68]. One year after the same study-group presented a single-stage expansion of the phase I trial exploring the drug in 50 ovarian cancer BRCA-mutated patients. Forty per cent of patients had a radiological or serological response, while the overall clinical benefit rate (CBR) was 46\%. Interestingly, for the first time, a correlation was reported between CBR and PFI; moreover, a post hoc analysis pinpointed an association of platinum sensitivity with Olaparib response both at radiological and serological level $(p=0.001$ and $p=0.002$ respectively) [69]. Despite the achievement of responses at a dose of $100 \mathrm{mg}$ twice daily, Audeh and colleagues conducted a proof-of concept phase II trial in order to compare objective response rate (ORR) of this dosage with the maximum tolerated dose of $400 \mathrm{mg}$ twice daily in a population of 57 heavily pretreated germline BRCA mutated (gBRCAm) ovarian cancer patients. ORR was $33 \%$ in the cohort treated with $400 \mathrm{mg}$ twice daily vs $13 \%$ in the cohort treated at the lower dose; the median progression free survival (PFS) was 5.8 months (95\% confidence interval CI 2.8-10.6) versus 1.9 months (95\% confidence interval CI 1.8-3.6) in favor of $400 \mathrm{mg}$ twice daily. The 
treatment at higher dose was well tolerated, with a slight increase in nausea, fatigue and anemia. Therefore $100 \mathrm{mg}$ bis in die (bid) dose seems to be less effective than $400 \mathrm{mg}$ bid. It must be noticed that allocation of patients was not randomized and the $100 \mathrm{mg}$ bid group had worse prognostic characteristics [70].

The better efficacy of the higher dose of $400 \mathrm{mg}$ bid was confirmed by the first randomized phase II trial, known as Study 12. The study enrolled 97 gBRCAm ovarian cancer patients relapsed within 12 months of previous platinum therapy and assigned them to receive Olaparib $200 \mathrm{mg}$ or $400 \mathrm{mg}$ continuously, both dose twice daily, or PLD $50 \mathrm{mg} / \mathrm{m} 2$ day $1 / 28$. Neither median PFS or ORR between Olaparib groups and PLD group reached statistical significance [71].

Olaparib seems to be active also in BRCA wild type ovarian cancer, as demonstrated for the first time in 2011 by Gelmon in a phase II trial, with a ORR of $24 \%$ versus the $41 \%$ reported in the gBRCAm patients [72]. These data confirmed the hypothesis that HRD and consequent susceptibility to platinum compounds or other drugs that create DNA damage, like PLD, or prevent damage repair, like PARPis, depend on various gene alteration and not only on BRCA mutation [28].

\subsection{Pivotal trials for Olaparib approval}

Olaparib monotherapy efficacy as a maintenance treatment was ratified in two randomized clinical trials, with more than 500 patients enrolled worldwide, known as Study 19 and SOLO 2, at the dose of $400 \mathrm{mg}$ twice daily (capsules) and $300 \mathrm{mg}$ twice daily (tablets), respectively.

In 2012 Lederman and colleagues [73] presented the first results of a double-blind, placebocontrolled phase II trial that enrolled 265 platinum-sensitive ovarian cancer patients previously treated with two or more platinum based chemotherapies and a complete or partial response achieved to the last platinum therapy. Patients were randomized to receive @Lynparza capsules 400 mg twice daily or placebo and the stratifications were done by response to the last platinum compound (complete versus partial), time to progression on penultimate platinum line and ancestry (Jewish versus non-Jewish). The primary endpoint of this trial was investigator-assessed PFS. PFS was significantly prolonged in the Olaparib group, 8.4 versus 4.8 months and a hazard ratio (HR) 0.35 (95\% CI 0.25-0.49, $\mathrm{p}<0.00001$ ). The first analysis of overall survival (OS), a secondary endpoint, showed no difference, although a pre-planned subgroup analysis seemed to highlight an advantage for patients with a known BRCA mutation. These modest results led to a "dark period" for Olaparib development because no drugs authorities would approve it in absence of greater and certain benefit [74]. In order to develop a "target therapy" meanwhile data on germline BRCA mutation status were derived from a retrospective analysis and showed that $36 \%$ of the enrolled 
population (96 patients) were mutated, 53 in the Olaparib group (39\%) and 43 in the placebo group (33\%). This new information opened a new lucky era for Olaparib two years later, with the results of a preplanned-subgroup efficacy analysis based on BRCA status. The effect of Olaparib in BRCA mutated patients was greater and lead to a PFS of 11.2 months versus 4.3 months observed in BRCA wild type (HR 0.18, 95\% CI 0.10-0.31, p<0.00001). A significant, even if smaller, benefit was reported also for BRCA wild type treated with Olaparib compared to the placebo group (7.4 versus 5.5 months, HR $0.54,95 \%$ IC $0.34-0.85, \mathrm{p}=0.0075$ ) [75]. Nor in the second [75] neither in the third interim analysis [76] a significant difference in OS was reported and this was probably due to the high rate of crossover (23\%). In this phase II study Olaparib showed a good tolerability profile with a dose interruption rate of $36 \%$ versus $16 \%$ in the placebo group; the grade 3 or worse side effects reported were fatigue, nausea and anemia. In patients treated for two years or more $75 \%$ reported low-grade nausea, $56 \%$ experienced fatigue, $38 \%$ vomiting and $25 \%$ anaemia. $15 \%$ of the BRCA mutated patients continued Olaparib for at least 5 years.

Since preclinical data proved that Olaparib could increase the efficacy of DNA-damaging chemotherapy, in 2015 Oza and colleagues [77] investigated this hypothesis and published the results of a randomized, open-label, phase II study in which, 162 platinum-sensitive, recurrent ovarian cancer patients, pre-treated at least with three previous platinum based chemotherapy lines were randomized to receive a) Olaparib $200 \mathrm{mg}$ capsules twice daily from day 1 to 10 of a 21-day cycle in association with carboplatin AUC $4 \mathrm{mg} / \mathrm{ml} / \mathrm{min}$ and paclitaxel $175 \mathrm{mg} / \mathrm{m} 2$ on day $1 / 21$ followed by Olaparib monotherapy (400 mg capsules twice daily given every day until progression; or b) carboplatin AUC $6 \mathrm{mg} / \mathrm{ml} / \mathrm{min}$ and paclitaxel $175 \mathrm{mg} / \mathrm{m}$ on day $1 / 21$ followed by none maintenance therapy. BRCA status was known or retrospectively retrieved for 107 patients; 38\% were mutated and equally divided between the two arms of treatment. PFS was greater in the Olaparib arm, with a median of 12.2 versus 9.6 months (HR $0.51,95 \%$ CI $0.34-0.77, \mathrm{p}=0.0012$ ), with a highly regarded advantage in BRCA mutated patients (HR 0.21, 95\% CI 0.08-0.55, $\mathrm{p}=0.0015$ ). The most common grade 3 or higher side effects during the association phase were neutropenia (43\% versus $35 \%$ in the chemotherapy alone group) and anemia (9\% versus 7\%), whereas serious adverse events were more frequently reported in the chemotherapy alone group (21\% versus $15 \%)$.

The endorsement of Olaparib primary role to prolong PFS and time to first and second subsequent chemotherapy arrived with the publication of the randomized, double-blind, phase III trial conducted by Pujade-Lauraine et al. The SOLO-2 trial enrolled 295 gBRCAm patients and randomized them to receive Olaparib tablets $300 \mathrm{mg}$ twice daily versus placebo after a partial or complete response to the most recent platinum based chemotherapy. Also in this trial, all patients 
were pre-treated with two or more platinum containing chemotherapy, with a nearly $40 \%$ of highly pretreated (three or more previous line) in the Olaparib group. Investigator assessed-PFS, the primary endpoint, was longer in patients treated with @Lynparza, with a median of 19.1 versus 5.5 months (HR 0.30, 95\% CI 0.22-0.41, p<0.0001)[25]. The advantage was seen in all subgroups, also in the patients pretreated with bevacizumab. As reported in the poster session of ESMO 2017 Congress, the superiority is reported also in the most heavily pre-treated patients with no statistical significant interaction between number of prior lines of platinum-based therapy received and PFS benefit [78]. Olaparib met also two secondary endpoints, actually considered OS surrogates, the time to first and second subsequent therapy, with HR 0.28 (95\% CI 0.21-0.38, p<0.0001) and HR 0.37 (95\% CI 0.26-0.53, p<0.0001), respectively. Moreover, Olaparib had no significant detrimental effect on health-related quality of life (HRQoL) [25] [79]. The most frequently reported side effects of CTCAE were low grade nausea, asthenia, vomiting, abdominal pain and diarrhea in both groups. The most common adverse event of grade 3 or higher in the Olaparib group was anemia, with blood transfusion rate of $18 \%$ versus $1 \%$ in the placebo group, whereas neutropenia and thrombocytopenia of grade 3 or worse severity were similar in the two groups.

Olaparib demonstrated to be effective also as monotherapy in a single arm study conducted by Kaufman [80], well known as Study 42, where 298 patients with deleterious or suspected deleterious gBRCAm patients with different kind of tumors, including 193 ovarian cancer patients heavily pre-treated (median of prior chemotherapy lines was 5). The ORR was $34 \%$ with a median duration of response (DOR) of 7.9 months. Domchek et al. published additional data about EOC gBRCAm population of this trial, focusing on the 137 patients with measurable disease and pretreated with three or more chemotherapy lines. ORR in platinum-resistant tumors was 30\%; interestingly, although the median DOR was similar for platinum-sensitive and platinum-resistant, the platinum sensitive population achieved better outcome across all the endpoints [81].

Efficacy of Olaparib treatment, considering both ORR and DOR, was confirmed also by a pooled analysis on 300 gBRCAm EOC patients conducted by Matulonis and colleagues, with a similar and manageable safety profile in more heavily pretreated patients ( $\geq 3$ lines of prior chemotherapy) [82].

\subsection{Indication and dosage}

Olaparib (@Lynparza) has been approved by FDA and EMA as maintenance therapy for recurrent epithelial ovarian, fallopian tube or primary peritoneal cancer after the achievement of a complete or partial response to chemotherapy with a platinum compound in patients with a somatic or germline mutations in BRCA 1 or 2 genes. The results presented by Kauffman [80] in December 
2014 led to faster FDA approval to use olaparib as monotherapy in gBRCAm patients after at least three prior lines by.

As reported previously Olaparib is available both as capsules and tablets. Based on the different pharmacokinetics and biovailability is not possible to change from one formulation to another on a milligram-to-milligram basis. The approved dose for oral intake of @Lynparza is $300 \mathrm{mg}$ (two 150 $\mathrm{mg}$ tablets) twice daily, with or without food, for a total daily dose of $600 \mathrm{mg}$. If tablets formulation is not yet available, the recommended capsules dose is $400 \mathrm{mg}$ (eight $50 \mathrm{mg}$ capsules) twice daily [83]. The treatment should be carried on until disease progression or unacceptable toxicity [66].

\subsection{Current strategies of development}

The recruiting, or about to recruit, ongoing clinical trial summarized in Table 1 would answer to some fundamental still pending questions on PARPi, and consequently, on Olaparib use. First of all, since the outmost important aim is to increase the cure rate, we are looking for results of SOLO 1 trial, where Olaparib in BRCAm patients is administered after the first line chemotherapy. Secondly, while today maintenance seems to be the right way to use this compound, some ongoing trials would give us data about the best treatment sequence (chemotherapy upfront versus PARP-I followed by chemotherapy). Moreover, it is important to investigate efficacy and safety of the possible combinations with promising new therapies for EOC like immunotherapy and antiangiogenic agents. The first encouraging clinical data on association of Cediranib, an oral tyrosine kinase inhibitor of vascular endothelial growth factor (VEGF) receptor with Olaparib were reported by Liu et al. The combination arm (Olaparib $200 \mathrm{mg}$ twice daily associated with Cediranib $30 \mathrm{mg}$ daily) achieved longer PFS then the control arm with Olaparib monotherapy (17 months versus 9 months, $\mathrm{p}=0.005$ ) [84]. Lastly, we do not have data on rechallenge role in Olaparib pretreated patients.

\section{NIRAPARIB}

\subsection{Description}

Niraparib (@Zejula, TESARO Inc.), formerly known as MK-4827, is an orally active and potent PARP inhibitor. The chemical name of Niraparib is tosylate monohydrate is 2-\{4-[85]phenyl $\}-2 \mathrm{H}$ indazole 7-carboxamide 4-methylbenzenesulfonate hydrate (1:1:1). The molecular formula is C26H30N4O5S and it has a molecular weight of 510.61 amu (Figure 3). Niraparib solubility is pH independent below the $\mathrm{pKa}$ of 9.95 , with an aqueous free base solubility of $0.7 \mathrm{mg} / \mathrm{mL}$ to 1.1 
$\mathrm{mg} / \mathrm{mL}$ across the physiological pH range. Each @Zejula capsule contains 159.4 mg niraparib tosylate monohydrate equivalent to $100 \mathrm{mg}$ Niraparib free base as the active ingredient [86].

\title{
3.2. Pharmacokinetics
}

Niraparib pharmacokinetics and first-in-humans activity was assessed in the phase I trial by Sandhu et al. [87]. $300 \mathrm{mg} /$ day was established as the maximum tolerated dose.

Following a single-dose administration of $300 \mathrm{mg}$ the mean $( \pm \mathrm{SD})$ peak plasma concentration (Cmax) was $804( \pm 403) \mathrm{ng} / \mathrm{mL}$. The systemic exposures (Cmax and AUC) of Niraparib increased in a dose proportional manner, with daily doses ranging from $30 \mathrm{mg}(0.1$ times the approved recommended dosage) to $400 \mathrm{mg}$ (1.3 times the approved recommended dosage).

\begin{abstract}
Absorption
The absolute bioavailability of Niraparib is approximately $73 \%$. Following oral administration of Niraparib, peak plasma concentration, Cmax, is reached within 3 hours. Concomitant administration of a high fat meal (800-1,000 calories) does not significantly affect the pharmacokinetics.
\end{abstract}

\section{Distribution}

Niraparib is $83.0 \%$ bound to human plasma proteins. The average $( \pm S D)$ apparent volume of distribution $(\mathrm{Vd} / \mathrm{F})$ was $1220( \pm 1114) \mathrm{L}$.

\section{Elimination}

Following multiple daily doses of $300 \mathrm{mg}$ Niraparib, the mean half-life (t1/2) is 36 hours. In a population pharmacokinetic analysis, the apparent total clearance (CL/F) was $16.2 \mathrm{~L} / \mathrm{h}$.

\section{Metabolism}

Niraparib is metabolized primarily by carboxilesterase (CEs) to form a major inactive metabolite, which subsequently undergoes glucuronidation.

\section{Excretion}

Following administration of a single oral $300 \mathrm{mg}$ dose Niraparib, the average percent recovery of the administred dose over 21 days was $47.5 \%$ (range $33.4 \%$ to 60.2\%) in urine, and $38.8 \%$ (range $28.3 \%$ to $47.0 \%$ ) in feces. 


\subsection{Clinical activity}

The activity and safety of Niraparib monotherapy $300 \mathrm{mg}$ once daily was first explored in the phase I trial by Sandhu and colleagues. Dose-limiting toxic effects were grade 3 fatigue, grade 3 pneumonitis, and grade 4 thrombocytopenia. Common treatment-related toxic effects were anaemia (48\%), nausea (42\%), fatigue (42\%), thrombocytopenia (35\%), anorexia (26\%), neutropenia (24\%), constipation (23\%), and vomiting (20\%), and were predominantly grade 1 or 2 . Pharmacodynamic analyses confirmed PARP inhibition exceeded $50 \%$ at doses greater than $80 \mathrm{mg} /$ day and antitumour activity was documented beyond doses of $60 \mathrm{mg} /$ day. Antitumor activity beyond HGSOC was also reported in non-small-cell lung cancer and prostate cancer [87]. Trial 1 (ENGOT-OV16/NOVA) was the first phase III trial designed and has studied Niraparib efficacy in 367 patients with platinum-sensitive recurrent ovarian, fallopian tube, and primary peritoneal cancer in [27]. The NOVA trial was a double-blind, placebo-controlled trial in which EOC patients $(n=553)$ were randomized 2:1 to Niraparib $300 \mathrm{mg}$ orally daily or matched placebo within 8 weeks of the last therapy. All patients had received at least two prior platinum-containing regimens and were in response (complete or partial) to their most recent platinum-based regimen. Randomization was stratified by time to progression after the penultimate platinum therapy ( 6 to $<12$ months and $\geq 12$ months), use of bevacizumab in conjunction with the penultimate or last platinum regimen (yes/no) and best response during the most recent platinum regimen (complete versus partial response). Eligible patients were assigned to one of two cohorts based on the results of the BRACAnalysis CDx. Patients with deleterious or suspected deleterious germline BRCA mutations (gBRCAm) were assigned to the germline $B R C A$ mutated (gBRCAmut) cohort ( $\mathrm{n}=203)$, and those without germline $B R C A$ mutations were assigned to the non-gBRCAmut cohort $(\mathrm{n}=350)$. The major efficacy outcome measure, PFS, was determined primarily by central independent assessment per RECIST (Response Evaluation Criteria in Solid Tumors, version 1.1). Results showed that PFS in the Niraparib group was significantly longer than in the placebo group in all three primary efficacy populations $(\mathrm{P}<0.001)$. In the $\mathrm{g} B R C A$ cohort, median PFS was 21.0 months in the Niraparib group and 5.5 months in the placebo group (HR 0.27). Moreover, Niraparib treatment resulted in significantly longer PFS in both the HRD-positive subgroup of the non-gBRCA cohort (median 12.9 months vs. 3.8 months) and in the overall non-gBRCA cohort (median 9.3 months vs. 3.9 months). Adverse reactions in the NOVA trial led to dose reduction or interruption in $69 \%$ of patients, most frequently for thrombocytopenia (41\%) and anemia (20\%). Analyses of patient-reported outcomes (PRO) indicated similar outcomes for those receiving Niraparib and those receiving placebo. The permanent discontinuation rate due to adverse reactions was about $15 \%$. The median exposure to Niraparib in this cohort was 250 days. 
Following these results several post hoc and subgroups analyses have already been done. First of all, Matulonis and colleagues analyzed data from the NOVA trial to determine the long-term efficacy of Niraparib and its impact on subsequent therapy [88]. Results showed that Niraparib was associated with a superior estimated PFS probability at 12, 18, and 24 months, regardless of patient's BRCA mutation status, suggesting that patients who had received treatment with Niraparib were not resistant to subsequent therapy. Secondary, Mirza et al. presented results of a post hoc analysis of efficacy, safety, and PRO from a subset of patients in the NOVA trial who achieved a PR after their last platinum-based chemotherapy $(n=272)$ [89]. A comparison of the PFS and Quality of life (QoL) HRs showed no difference between the overall population and patients who achieved a PR to their most recent platinum-based chemotherapy. Moreover, Dr. José Del Campo and colleagues evaluated platinum sensitivity and outcomes in patients assigned to the placebo arm in the NOVA trial [90]. Patients who experienced disease progression within 6 months after their last platinum-based regimen had received more prior lines of platinum-based therapy, as well as more lines of any chemotherapy, compared with patients whose remission lasted 6 months or longer. Among patients with germline BRCA1/2 mutation, 46\% of those progressing within 6 months and 39\% of those progressing at 6 months or later received 3 or more lines of platinumbased therapy, and $63 \%$ vs $45 \%$, respectively, received 3 or more lines of any type of chemotherapy. Among BRCA1/2 wildtype patients, $29 \%$ of those who progressed within 6 months and $15 \%$ of those who progressed at 6 months or later received 3 or more lines of platinum-based therapy, and $43 \%$ vs 19\%, respectively, received at least 3 lines of any chemotherapy, indicating that a substantial number of patients with platinum resistant ovarian cancer was included in the NOVA trial. Finally, more recently Mirza at the European Society of Gynecologic Oncology Meeting 2017 presented data showing how in the NOVA trial thrombocytopenia grade 3-4 correlated with patients weight (if $<67 \mathrm{~kg}$ ) and myelosuppression during the prior chemotherapeutic regimen[91]. These data belong to a retrospective analysis, thus further investigations are required to better tailor Niraparib dosage in the real clinical practice setting.

\subsection{Indication and dosage}

Following the results of the NOVA trial [27] Niraparib (@Zeyula) received both FDA and EMA approval as maintenance treatment of adult patients with recurrent EOC, fallopian tube, or primary peritoneal cancer in a complete or partial response to platinum-based chemotherapy, regardless BRCA status and number of previous lines of treatment. The recommended dose is $300 \mathrm{mg}$ once daily. Patients should start treatment within 8 weeks after their most recent platinum-containing regimen and treatment should be continued until disease progression or unacceptable toxicity [86]. 


\subsection{Current strategies of development}

Niraparib combinations with molecular-targeted agents and cytotoxic agents are under investigation and in some cases have moved to the clinical trial phase. Several clinical trials are actually ongoing both in platinum-sensitive and platinum-resistant setting, investigating the activity of Niraparib single agent as first line therapy or Niraparib in association with anti-PD1 and anti-VEGF agents (Table 2).

\section{RUCAPARIB}

\subsection{Description}

Rucaparib (@Rubraca, Clovis) also known as AG-014699 and PF-01367338 is an oral PARP-1, 2 and 3 inhibitor. Its molecular formula is C19H18FN3O and its chemical name is 8-fluoro-2-(4((methylamino)methyl)phenyl)-4,5-dihydro -1h-azepino [5,4,3-cd]indol-6(3H)-one. The molecular mass of Rucaprib is 555.67 Daltons. As previously reported this drug differs by others PARP inhibitors because of its inhibitory activity also on other enzymes involved in homologous chromosomal recombination called tankyrases [63]. The chemical structure of Rucaparib is reported on Figure 4 [92].

\subsection{Pharmacokinetics}

The pharmacokinetics, safety and activity of oral Rucaparib were evaluated in 56 patients, primarily affected by breast or ovarian cancer, enrolled by Kristelet et al in a phase I/II trial (Study 1) [93].

Absorption The median time to reach maximum concentration (Cmax) is 1.9 hours at the approved dosage. The mean absolute bioavailability of Rucaparib immediate-release tablet is $36 \%$ with a range from $30 \%$ to $45 \%$. Following a high-fat meal, the Cmax increases by $20 \%$, and the time to reach C max is prolonged by 2.5 hours [92] [94].

Distribution The protein binding of Rucaparib observed in vitro was $70 \%$ in human plasma at therapeutic concentrations. Rucaparib preferentially distributed to red blood cells with a blood-toplasma concentration ratio of 1.83 .

Elimination The mean terminal half-life after a single oral dose of Rucaparib ranges from 17 to 19 hours. The apparent clearance ranges from 15.3 to $79.2 \mathrm{~L} /$ hour with $600 \mathrm{mg}$ Rucaparib twice daily. 
Metabolism Rucaparib is metabolized by CYP2D6 and to a lesser extent by CYP1A2 and CYP3A4.

Excretion Rucaparib is excreted mainly through the faeces, accounting for $>=79 \%$ of the total dose.

\subsection{Clinical activity}

The efficacy of Rucaparib in monotherapy for the treatment of EOC was first assessed in two multicentre single arm trial, the phase I/II study, named Study 1 (NCT01482715) and the phase II trial Study 2 (ARIEL2). In the first part of study 1, as previously reported, 56 patients were enrolled and the $35,7 \%$ of them was affected by platinum sensitive or platinum resistant ovarian cancer. One patient with platinum sensitive EOC achieved a complete response and two patients with a platinum resistant EOC obtained a partial remission. No grade 4 adverse events were observed. The most common drug-related toxicities reported were fatigue, gastrointestinal disorders, myelotoxicity, hyporexia, and elevation of transaminase levels. In the second part of the trial authors evaluated the activity of Rucaparib in 42 patients with platinum sensitive ovarian cancer with BRCA1/2 germline mutations. This population reported an objective response rate of $59.5 \%$ and a median duration of response of 7.8 months (range 5.6-10.5) [93] [95].

In the first part of the phase II ARIEL 2 trial 204 patients with recurrent platinum sensitive EOC were enrolled and classified in three subgroups on the basis of HRD status (BRCA mutated ( $\mathrm{n}=40$ ), LOH high (n=82), LOH low (n=70). All patients were treated with $600 \mathrm{mg}$ of oral Rucaparib twice daily. A median PFS of 12.8, 5.7 and 5.2 months was observed respectively in the three subgroups. The difference observed between patients harboring BRCA mutation or with LOH high was significantly greater than in the low LOH group. The most common grade 3 or worse toxicities observed were anemia and transaminase increase [26]. The second part of ARIEL 2 trial is still ongoing; 286 patients who have received at least 3 lines of chemotherapy with both platinum sensitive or platinum resistant disease have been enrolled to evaluate clinical activity of Rucaparib in this different setting based on HRD status. In another phase II trial Drew et al investigated intermittent and continuous schedules of intravenous or oral Rucaparib in 78 patients with advanced breast or ovarian cancer harbouring BRCA1/2 mutations. The ORR for oral Rucaparib (across all dose levels) was 15\%. 12 out of 13 patients treated with continuous oral Rucaparib obtained a CR/PR or SD for more than 12 weeks, with a median duration of response of 179 days. The intermittent intravenous Rucaparib resulted in an ORR of only $2 \%$. However, $41 \%$ of patients achieved SD for longer than 12 weeks, with 3 patients maintaining disease stabilisation for more 
than 52 weeks. All the 51 patients with ovarian cancer enrolled in this study have been previously treated with a platinum-based chemotherapy; authors observed a correlation between response rate to Rucaparib and PFI. No response was reported among breast cancer patients. The half-life of Rucaparib after oral dosing reported in this study was $9.1 \pm 2.7$ hours. The most common reported adverse events were fatigue and nausea [96].

The results of the phase III trial ARIEL 3 have been recently published. In this randomized, doubleblind, study 564 patients with platinum sensitive EOC in response after their last platinum-based chemotherapy were treated with Rucaparib $600 \mathrm{mg}$ twice daily or placebo. Authors reported a prolonged PFS in patients assigned to the experimental arm. For BRCA mutated patients median PFS was respectively of 16.6 months in the Rucaparib arm versus 5.4 months in the placebo group. A significant increase in PFS was also observed in patients without BRCA mutation, PFS reported for patients with a HRD carcinoma treated with Rucaparib was 13.6 months vs 5.4 for the placebo group while in the intention to treat population was 10.8 versus 5.4 months. Grade 3-4 adverse events were more frequent in the experimental arm (56\% vs 15\%) and the most common were anaemia and transaminase elevation [44].

\subsection{Indication and dosage}

Rucaparib have been approved by the FDA as a single agent for the treatment of patients affected by relapsed ovarian cancer with germline or somatic BRCA mutations who have been treated with two or more lines of chemotherapy. The recommended phase II dose of single-agent oral Rucaparib is 600mg twice daily [93]. Based on the results of ARIEL 3 trial, Clovis Oncology have recently submitted a supplemental New Drug Application (sNDA) for a second-line and later maintenance treatment indication for patients with recurrent platinum-sensitive EOC in response to their most recent platinum therapy

\subsection{Current strategies of development}

Rucaparib is actually the only PARP inhibitor approved in monotherapy regardless of platinum sensitivity, following the publication of the results of Study 1 and ARIEL 2 demonstrating the efficacy in monotherapy in patients with recurrent BRCA mutated EOC. Considering the fewer available options of treatment for patients with platinum resistant disease NCCN guidelines recommends Rucaparib single agent also in this setting in presence of BRCA mutations [97]. As previously reported it is feasible that early Rucaparib will also be approved as maintenance therapy in patients with platinum sensitive relapsed EOC in response to their last platinum-based 
chemotherapy. Several trials investigating the activity of Rucaparib, both as a single agent and after platinum based chemotherapy for treatment of ovarian cancer, are ongoing. A phase I study evaluating the association of Rucaparib and the anti-PD-L1 Atezolizumab is actually recruiting patients. The others ongoing clinical trials are summarized in Table 3.

\section{TALAZOPARIB}

\subsection{Description}

Talazoparib (also known as MDV3800 and BMN 673) is a selective inhibitor of PARP1 and2. The molecular formula of Talazoparib is $\mathrm{C} 19 \mathrm{H} 14 \mathrm{~F} 2 \mathrm{~N} 6 \mathrm{O}$ and its chemical name is (8S,9R)-5-fluoro-8(4-fluorophenyl)-9-(1-methyl-1H-1,2,4-triazol-5-yl)-8,9-dihydro-2H-pyrido[4,3,2-de]phthalazin$3(7 \mathrm{H})$-one. The molecular weight is $380.359 \mathrm{~g} / \mathrm{mol}$. The chemical structure of Talazoparib is shown in Figure5.

\subsection{Pharmacokinetics}

The antitumor activity and pharmacokinetics of Talazoparib was evaluated for the first time by De Bono et al in a 2-stage dose-escalation trial [98]. In the first part of this study 39 patients with BRCA mutated tumors were enrolled in 9 cohorts from 25 to $1100 \mu \mathrm{g} / \mathrm{d}$. The maximum tolerated dose was defined at $1000 \mu \mathrm{g} / \mathrm{d}$. An inhibitory activity against PARP was reported at doses $\geq 100$ $\mu \mathrm{g} / \mathrm{day}$ in PBMCs. Steady state plasma concentrations were reached by the end of the second week of daily dosing; the maximum plasma concentration was reached within 2 hours after all evaluated doses .

\section{Absorption}

In a subsequent dose-escalation phase I study [99] conducted in patients with BRCA 1 or 2 mutations Talazoparib has shown to have a rapid absorption. The maximum plasma concentration (Cmax) was reached within 2 hours after all evaluated doses.

\section{Distribution}

Talazoparib has demonstrated to be well distributed into tissues, and its volume of distribution seems to be in excess of the volume of the systemic circulatory space.

\section{Elimination}

The half-life observed at the MTD dose of $1.0 \mathrm{mg} /$ day was nearly 48 hours. 


\section{Excretion}

Results for urinary elimination of the parent compound suggest linear urinary elimination kinetics after daily Talazoparib dosing between the 0.025 and $1.1 \mathrm{mg}$ dose levels

\subsection{Clinical activity}

The evaluation of Talazoparib activity in the treatment of ovarian cancer is still at an early stage. In 2016 Dhawan et al. presented the results of a phase I/II trial evaluating Talazoparib in combination with carboplatin in patients with solid tumors with or without germline mutations. Among the 24 patients enrolled, 2 patients (8\%) were affected by EOC and $20 \%$ were BRCA1/2 mutant cases. Other grade $3 / 4$ toxicities reported were fatigue (13\%), neutropenia (63\%), thrombocytopenia (29\%), and anemia (38\%). A deeper decrease of the neutrophil count was observed in patients with germline BRCA mutations. Authors reported $1 \mathrm{CR}$ and 2 PR among BRCA mutated patients. Four patients maintained a stable disease beyond 4 months; 3 of them had a somatic BRCA mutation and the fourth had a BRIP germline [100].

De Bono et al investigated the safety pharmacokinetics and activity of Talazoparib in a phase I/II doseescalation trial enrolling patients with germline BRCA1 or 2 mutated tumors or selected patients with sporadic cancers. A sustained PARP inhibition was observed at doses $\geq 0.60 \mathrm{mg} / \mathrm{day}$. The most common possibly related adverse event observed were fatigue; nausea; anemia; neutropenia; thrombocytopenia and grade 1 alopecia. A dose-limiting thrombocytopenia was observed respectively in $1 / 6$ and $2 / 5$ patients at 900 and $1100 \mu \mathrm{g} / \mathrm{d}$. In this trial 34 EOC patients were enrolled, 25 of them with germline BRCA mutated, all previously treated with platinum based chemotherapy were enrolled, 12 of them with measureable disease. Among them $42 \%$ had an objective response rate and $67 \%$ add a clinical benefit rate with a median PFS of 36.4 weeks [99].

Results from phase II or III in patients with EOC are not available. However regarding patients with BRCA mutations encouraging signs about the efficacy of Talazoparib came from a phase II study conducted in patients with BRCA positive breast cancer (ABRAZO) in which an ORR of $24 \%$ and $34 \%$ respectively was reported for BRCA 1 and BRCA 2 mutations carriers [101].

\subsection{Current strategies of development}

Evidences supporting the use of Talazoparib in the treatment of EOC are few in comparison with other PARPis. However some studies evaluating the activity of Talazoparib in ovarian carcinoma are ongoing (Table 4), including a phase 1 study in association with a checkpoint inhibitor and a 
phase II study exploring Talazoparib activity in advanced cancers with BRCA1/2 germline or somatic alterations, PTEN mutations or PTEN loss and HRD defect.

\section{VELIPARIB}

\subsection{Description}

Veliparib, also called ABT-888, is a poly(ADP-ribose) polymerase (PARP) -1 and -2 inhibitor with chemosensitizing and antitumor activities. Veliparib has no antiproliferative effects as a single agent. The molecular formula of Veliparib is C13H16N4O and its IUPAC name is 2-[(2R)-2methylpyrrolidin-2-yl]-1H-benzimidazole-4-carboxamide. The chemical structure of Veliparib is reported in Figure 6.

\subsection{Pharmacokinetics}

The pharmacokinetics of Veliparib was assessed by Kummar et al in phase 0 trial. They observed that the absorption peak occurs after 0.5-1.5 hours with a single dose of $50 \mathrm{mg}$ [102]. In a phase I trial presented by Puhalla et al in 2014 the half-life of Veliparib was assessed at 5.2 hours [103].

\section{Absorption}

Veliparib demonstrated to have a good oral bioavailability, and a peak absorption between 0.5 and 1.5 hours, with a $\mathrm{C}$ max of $0.45 \mu \mathrm{M}$ after a single dose of $50 \mathrm{mg}$.

\section{Distribution}

Significant inhibition of PARP levels in both tumor tissue and peripheral blood mononuclear cells was observed 3-6 hours after administration

\section{Metabolism and Excretion}

Veliparib is excreted previously in the urine; however, in a lower percentage (nearly 13\%) Veliparib undergoes hepatic metabolism by the activity of cytochrome CYP2D6.

\subsection{Clinical activity}


In 2014 Puhalla et al. presented the results of a phase I trial aimed at assessing maximum tolerated dose, dose-limiting toxicities, pharmacokinetic and pharmacodynamic properties, and preliminary efficacy of Veliparib. Eighty-eight patients, mainly affected by ovarian or breast cancer, were enrolled; 60 were BRCA positive and 28 BRCA wild type. The recommended phase 2 dose was $400 \mathrm{mg}$ BID. The most common toxicities reported were nausea, fatigue, and lymphopenia. The half-life was established at 5.2 hours. BRCA mutated patients had an ORR of $23 \%$ and a clinical benefit rate (CBR; $\mathrm{CR}+\mathrm{PR}+$ stable disease) of 58\% across all dose levels. Among BRCA-wild type patients the ORR was $4 \%$ with a CBR of $38 \%$. Coleman et al. evaluated the activity of Veliparib in a phase II trial in 52 BRCA mutated patients with persistent or recurrent ovarian cancer. Sixty percent of patients was platinum resistant. Veliparib was administered at $400 \mathrm{mg}$ orally twice a day. No drug related deaths were reported. The most common toxicities observed were mielotoxicity and gastrointestinal toxicity. Authors reported a median PFS of 8.11 months (ranging from 0.43 to 19.55 months) and a median OS of 19.7 months (ranging from 2.3 to 19.7 months) [104].

Recently, the results of a phase I/II trial have been published exploring the role of Veliparib monotherapy in patients with germline BRCA mutations platinum sensitive or resistant ovarian cancer. Sixteen patients were enrolled in the phase I study, and 32 in the second part of the study. The maximum tolerated dose was established at $300 \mathrm{mg}$ twice daily. Median PFS and OS for the intention to treat population were respectively 5.6 and 13.7 months. The most common toxicities observed were fatigue, nausea and vomiting [105]. In another phase I study Nishio et al evaluated the safety, tolerability, pharmacokinetics and efficacy of Veliparib (100 or 150 twice a day) in association with Carboplatin and weekly Paclitaxel in 9 Japanese patients with newly diagnosed EOC. The most frequent toxicities were neutropenia, alopecia, neurotoxicity, anemia and nausea. Authors reported an ORR of $100 \%$ in the 5 patients evaluated (the other 4 had no measurable lesions). After this study the dose of Veliparib in association with Carboplatin and Paclitaxel was established at $150 \mathrm{mg}$ twice a day [106].

The safety of this PARP inhibitor was also assessed in association with chemotherapy (PLD, Carboplatin and Bevacizumab) in a phase I trial enrolling patients with platinum sensitive ovarian cancer. DLTs reported in the dose escalation part included grade 4 thrombocytopenia and prolonged neutropenia. With the addiction of bevacizumab, a DLT was observed in 9 out of 12 patients and these included grade 4 thrombocytopenia, prolonged neutropenia, hypertension and sepsis. The maximum tolerated dose of Veliparib in association with carboplatin was established at $80 \mathrm{mg}$ twice a day [107]. Kummar et al conducted a phase II study comparing low dose Cyclophosphamide 
alone or in association with Veliparib in patients with ovarian cancer. Seventy two patients were enrolled; no differences were reported for the two arms of treatment in terms of response rate [108].

In a phase I trial Reiss et al. evaluated the activity of Veliparib combined with low-dose fractionated whole abdominal radiation therapy in 32 patients with carcinomatosis due to advanced solid tumors. Eighteen patients were affected by EOC, 5 of them were BRCA mutations carriers. One objective response was observed, and stable disease was reported in $33 \%$ of patients. Patients with BRCA mutated EOC had a PFS of 4.47 months compared to PFS 3.58 months in the nonBRCA carriers and an OS of 10.15 months compared to 7.89 months in non-BRCA carriers. Patients whit platinum-sensitive disease had a PFS of 7.92 months compared to 3.58 months in platinum-resistant EOC. The most frequently observed toxicity was myelosuppression [109].

\subsection{Current strategies of development}

As discussed above, Veliparib alone has no anti-proliferative effect. However, there are several preclinical and clinical evidences of its activity as sensitizing agents for DNA-damaging treatments, such as chemotherapy and radiotherapy. As previously reported for Talazoparib, evidences supporting the use of Veliparib in the treatment of EOC are fewer compared to those for other PARP inhibitors, however several studies are ongoing, both with Veliparib as a single agent and in association with chemotherapy, including a phase III trial evaluating Veliparib as first line treatment in association with carboplatin and paclitaxel (Table 5). This randomized study includes three treatment arms: one with chemotherapy only, the second with chemotherapy followed by Veliparib in maintenance and the third with Veliparib associated with chemotherapy followed by Veliparib as a maintenance. Considering as primary outcome PFS, an enrolment of 1100 patients is estimated. There is also a phase II trial evaluating Veliparib as a single agent for the treatment of relapsed EOC (Table 5). Fifty-one patients are expected to be enrolled in this trial, patients with both platinum sensitive and platinum resistant disease are eligible but they must have a germline BRCA1/2 mutation.

\section{CURRENT AND FUTURE DEVELOPMENTS}

The development of PARPis is certainly the most important breakthrough in the treatment of ovarian cancer. Both FDA and EMA have already changed clinical practice after Olaparib and Niraparib approvals, but several questions regarding their use, strongly connected each other, still need an answer. 
1) Which is the optimal clinical setting?

2) How can we choose among different PARPis?

3) How can we better identify HRD patients?

4) How much do we know about resistance to PARPis?

5) Is it better to use PARPis alone or in association with other agents?

1) Even thought the activity of PARPis as maintenance therapy has been demonstrated [25] [27] [44] ongoing trials are seeking to clarify the ideal time for these agents to be used in the course of ovarian cancer. Results from SOLO-1, ARIEL-4, PRIMA and QUADRA trials will help to answer this question (for the details see Table 1,2 and 3). The first three trials aim to demonstrate that an early maintenance with PARPis after the frontline treatment could improve the outcome, while QUADRA trial is investigating chemotherapy free-regimen in heavily pretreated patients. Indeed, even if Olaparib activity has already been investigated in the above mentioned setting by Kaufman et al. [80], only FDA has recognized this indication. Moreover, as reported above, during the poster session of ESMO 2017 Congress, Olaparib superiority in SOLO-2 study is reported also in heavily pre-treated patients, with no difference of efficacy regarding number of prior lines of platinumbased therapy received [78]. Further data, regardless the BRCA status, are looked-for in Europe, in order to potentially extend the use of PARPis, first of all Niraparib, in poor setting as platinum resistant disease. Moreover Olaparib is currently under investigation in the neoadjuvant setting in the NEO trial.

2) At first relapse, BRCA mutated platinum sensitive recurrence ovarian cancer should go for secondary cytoreductive surgery, if a complete resection is expected [110]. If patients are not eligible for surgery, they should receive a platinum doublet followed by maintenance therapy, either with Bevacizumab, or with any PARPis, in case of CR or PR. However the difference in terms of PFS is strongly in favor of PARPis. On the contrary, in BRCA WT, PFS results from Bevacizumab and Niraparib (the only PARPi for relapsed BRCA WT patients) are similar, and therefore, the choice should be based on toxicities and disease characteristics. For example Bevacizumab seems more active, in patients with bulky disease, ascites and high Ca125 [111], but it may lead to hemorrhage and thrombosis more frequently than Niraparib. Beyond their activity, each PARPi shows different toxicities that can drive clinical choices, particularly in a maintenance setting. Nausea and fatigue were the most common side effects, reported in about $50 \%$ of patients with $10 \%$ of G3 or G4 regardless of the type of PARPi [112]. Instead, 3\% of patients treated with Olaparib reported G3-G4 abdominal pain, while $8 \%$ of patients treated with Niraparib developed severe 
hypertension [113]. The percentage of hematologic adverse events varied depending on which PARPis were used. Indeed, while anemia is common in patients treated with Olaparib, Niraparib and Rucaparib, neutropenia and thrombocytopenia are typical side effect of Niraparib (with rate of $20 \%$ and $33 \%$ respectively), while Rucaparib is characterized by $10 \%$ of asymptomatic increase of AST and ALT that normalizes over time [25] [27] [44]. Niraparib required a dose reduction in $66 \%$ of patients, while Olaparib in $54 \%$ and Rucaparib in $25 \%$ of patients nevertheless adverse events apparently do not affect quality of life neither in SOLO 2 nor in NOVA trial [114]. Thanks to these data, we can actually choose the best PARPi for each patient, considering residual toxicities, disease burden and BRCA status. Beside acute toxicities, potential long term toxicities are not clear and need careful surveillance. Preclinical model suggests a role of PARP-1 in cardiovascular diseases and long-term memory formation [112]. but, above all, an increased risk of MDS /AML was reported in patients treated with PARPis. It could be related to the high genomic instability of HRD patients (above all BRCA mutated patients) for whom further drugs induce DNA damage could raise this risk [112]. Currently, only few data are available on long-term exposure to Olaparib, that reported no warning on long-term toxicities [76], but further information, especially on Rucaparib and Niraparib, is needed. Moreover, we have no long term benefit predictors to PARPis, although an observational and sample collection study (A Study of Long-Term Responders on Olaparib, OLALA NCT02489058) is trying to identify them.

3) Even though germline BRCA mutations are the best predictors of PARPis activity in EOC, their efficacy has been observed also in patients without this alteration. Niraparib and Rucaparib have proved their activity also in HRD positive [27] [44], but different test to examine HRD status were used, as summarized in the introduction. While testing for BRCA mutations have already been incorporated into everyday clinical practice, a well recognized test to identify BRCAness phenotype due to a homologous recombination impairment does not exist yet [115]. Numerous patents have been registered regarding new methods trying to determinate HRD status (WO 2016138574 A1)[116], DNA sequences defects (WO 2017008912 A1)[117] or LOH (9388472)[118].

In the near future, the systematic evaluation of RAD51, $\gamma \mathrm{H} 2 \mathrm{AX}$ and Fanconi family of genes foci together with genomic instability might help to identify patient populations who can be classified as responders or non-responders to PARPis (for discussion of resistance mechanism see point 4 below)[119]. Early data in breast cancer have demonstrated a correlation between cytoplasmic PARP expression, detected by immunohistochemistry, a procedure sustainable and reproducible also in peripheral hospital, and high sensitivity to treatment [120]. Identification of responders is useful to optimize cost-benefit rate. Waiting for the genome profiling, actually the only parameters 
significantly associated with PARPis response, except BRCA status, is PFI, firstly identified by Fong in 2010 [69] and confirmed in a Cochrane's review including nearly 600 patients [121].

4) It is well known that even if a patient is BRCA mutant, after a long-term exposure to PARPis, develops drug resistance through different mechanisms [122]. The first mechanism studied has been the recovery of HRR ability, through secondary mutations restoring BRCA activity, as demonstrated by Norquist in a group of 46 recurred EOC. This retrospective analysis showed mutations were higher in platinum-resistant patients than in the sensitive ones $(46 \%$ versus $5 \%$, $\mathrm{p}=0.003$ ) [123]. Dhillon proposed the restoration of BRCA activity begins from BRCA-deficient and chemosensitive cells as a consequence of numerous mutations induced by platinum agents. This initial restored clone will expand during drug exposure-related selective pressure [124]. A recent patent by Yen et al. (US20170035737)[125] proposed a new method by administering COH29 and a DNA-damaging agent in a combined synergistic amount. In ARIEL 2 trial PARPis resistance seems to be related with secondary mutation of RAD51, one of the gene causing HRD when defective. If secondary mutations occur in RAD51C and RAD51D and their activity is recovered PARPis resistance arises [126]. Rottenberg and colleagues demonstrated in an in vivo model a possible resistance mechanism due to an increased expression of P-Glycoprotein (multi-drug resistance protein), a drug efflux pump responsible of a decrease intracellular drug concentration [127]. In 2015 Patch performed whole genome sequencing of nearly 100 ovarian cancers and reported various other acquired resistance mechanisms such as loss of BRCA1 promoter methylation and ABCB1 rearrangement, but their clinical impact are already unknown [128].

5) As already described several PARPis associations with molecular-targeted agents, standard cytotoxic agents and/or immunotherapy are currently under investigation and in some cases have already moved to the clinical trial phase (for complete list of ongoing trials see the above tables). While Olaparib and Niraparib have been approved as single-agents maintenance therapy, Veliparib seems to be the best candidate for chemotherapy-associations due to its toxicity profile. Several patents have recently been published exploring PARPIs combinationations with chemotherapy, immune-agents, TKIs or small nucleic acid molecule (e.i patents n. WO/2017/029517A1, US20170035737, US20170049767, WO/2017/151554A1, US20170100368, WO 2016209935 A1, US20170049767, 9707302, WO/2017/151554A1，WO/2017/013237A1，WO/2017/029517A1)[125, 129131]. Interestingly, Blanchette et al. registered the combination between PARPI, a topoisomerase-1 inhibition and irinotecan (WO/2017/031442A1)[132]; the topoisomerase-1 inhibitor can be delivered as a liposomal formulation resulting in an increased efficacy of the PARP inhibitor and reduced toxicity of the irinotecan. 
Moreover, a phase I study of Veliparib + whole abdomen radiotherapy has already shown efficacy with a 33\% rate of stable disease [109] suggesting its potential role also as chemo-sensitizer. On the other hand, no definitive data sustain a potential role of RT in increasing systemic PARPis response (the well-known abscopal effect) [133] [134]. A robust rationale exists instead on the association of PARPis with immunotherapy. BRCA mutant patients show a higher mutational load and number of neoantigens than BRCA WT, which reflects in an increased recruitment of tumor infiltrating lymphocytes (TILs) and potential response to checkpoints inhibitors[135] [136] [137]. In this field, Lambrechts et al. registered a patent (WO 2013153130 A1) [138]to detect mismatch repair (MMR) deficient tumors and exploring the synergism between PARPIs and MMR.

There is also strong pre-clinical evidence that support combination with anti-angiogenic agents. In fact the inhibition of VEGF induces chronic hypoxia leading to down-regulation of DNA repair mechanisms and creating an artificial HRD status which increase sensitivity to PARPis [84] [139]. AVANOVA, OCTOVA and CONCERTO are three examples of active trials exploring this setting.

For an exhaustive update on patents exploring PARPIs in ovarian cancer see Table 6.

Finally, a good opportunity to study new drugs and combinations as soon as they are developed is to create common bio-banks of tumor tissues as established in phase II ENGOT (NCT03267589) a cooperative innovative trial.

\section{CONCLUSION}

PARP inhibition has already proved to be an outstanding strategy for improving PFS in ovarian cancer, although a longer follow-up is needed to assess also OS benefit. The development of new combinations through clinical trials and new patents will provide a better understanding of PARPIs mechanisms of action and hopefully reflects in better clinical benefit for patients. 
Ethics Approval and Consent to Participate

Not applicable.

Human and Animal Rights

No Animals/Humans were used for studies that are base of this research.

Consent for Publication

Not applicable.

Conflict of Interest

All authors have no conflict of interest to declare.

Acknowledgements 


\section{REFERENCE LIST}

[1] Bethesda. (2016, 20 March 2017). http://seer.cancer.gov/csr/1975_2013/.

[2] J. A. Ledermann et al., "Newly diagnosed and relapsed epithelial ovarian carcinoma: ESMO Clinical Practice Guidelines for diagnosis, treatment and follow-up," (in eng), Ann Oncol, vol. 24 Suppl 6, pp. vi24-32, Oct 2013.

[3] A. Gadducci et al., "Are surveillance procedures of clinical benefit for patients treated for ovarian cancer?: A retrospective Italian multicentric study," (in eng), Int J Gynecol Cancer, vol. 19, no. 3, pp. 367-74, Apr 2009.

[4] G. E. Konecny and R. S. Kristeleit, "PARP inhibitors for BRCA1/2-mutated and sporadic ovarian cancer: current practice and future directions," (in eng), Br J Cancer, vol. 115, no. 10, pp. 11571173, Nov 2016.

[5] T. Lindahl, "Instability and decay of the primary structure of DNA," (in eng), Nature, vol. 362, no. 6422, pp. 709-15, Apr 1993.

[6] J. H. Hoeijmakers, "Genome maintenance mechanisms for preventing cancer," (in eng), Nature, vol. 411, no. 6835, pp. 366-74, May 2001.

[7] J. M. Lee, J. A. Ledermann, and E. C. Kohn, "PARP Inhibitors for BRCA1/2 mutation-associated and BRCA-like malignancies," (in eng), Ann Oncol, vol. 25, no. 1, pp. 32-40, Jan 2014.

[8] J. Rodon, M. D. Iniesta, and K. Papadopoulos, "Development of PARP inhibitors in oncology," (in eng), Expert Opin Investig Drugs, vol. 18, no. 1, pp. 31-43, Jan 2009.

[9] R. Plummer, "Perspective on the pipeline of drugs being developed with modulation of DNA damage as a target," (in eng), Clin Cancer Res, vol. 16, no. 18, pp. 4527-31, Sep 2010.

[10] M. E. Moynahan and M. Jasin, "Mitotic homologous recombination maintains genomic stability and suppresses tumorigenesis," (in eng), Nat Rev Mol Cell Biol, vol. 11, no. 3, pp. 196-207, Mar 2010.

[11] F. Aymard et al., "Transcriptionally active chromatin recruits homologous recombination at DNA double-strand breaks," (in eng), Nat Struct Mol Biol, vol. 21, no. 4, pp. 366-74, Apr 2014.

[12] S. K. Radhakrishnan, N. Jette, and S. P. Lees-Miller, "Non-homologous end joining: emerging themes and unanswered questions," (in eng), DNA Repair (Amst), vol. 17, pp. 2-8, May 2014.

[13] Y. Ma, H. Lu, K. Schwarz, and M. R. Lieber, "Repair of double-strand DNA breaks by the human nonhomologous DNA end joining pathway: the iterative processing model," (in eng), Cell Cycle, vol. 4, no. 9, pp. 1193-200, Sep 2005.

[14] M. R. Lieber, "The mechanism of double-strand DNA break repair by the nonhomologous DNA endjoining pathway," (in eng), Annu Rev Biochem, vol. 79, pp. 181-211, 2010.

[15] L. S. Friedman et al., "Confirmation of BRCA1 by analysis of germline mutations linked to breast and ovarian cancer in ten families," (in eng), Nat Genet, vol. 8, no. 4, pp. 399-404, Dec 1994.

[16] Y. Miki et al., "A strong candidate for the breast and ovarian cancer susceptibility gene BRCA1," (in eng), Science, vol. 266, no. 5182, pp. 66-71, Oct 1994.

[17] R. Wooster et al., "Identification of the breast cancer susceptibility gene BRCA2," (in eng), Nature, vol. 378, no. 6559, pp. 789-92, 1995 Dec 21-28 1995.

[18] A. Antoniou et al., "Average risks of breast and ovarian cancer associated with BRCA1 or BRCA2 mutations detected in case Series unselected for family history: a combined analysis of 22 studies," (in eng), Am J Hum Genet, vol. 72, no. 5, pp. 1117-30, May 2003.

[19] S. Chen and G. Parmigiani, "Meta-analysis of BRCA1 and BRCA2 penetrance," (in eng), J Clin Oncol, vol. 25, no. 11, pp. 1329-33, Apr 2007.

[20] P. A. Konstantinopoulos, R. Ceccaldi, G. I. Shapiro, and A. D. D'Andrea, "Homologous Recombination Deficiency: Exploiting the Fundamental Vulnerability of Ovarian Cancer," (in eng), Cancer Discov, vol. 5, no. 11, pp. 1137-54, Nov 2015.

[21] J. Prat, A. Ribé, and A. Gallardo, "Hereditary ovarian cancer," (in eng), Hum Pathol, vol. 36, no. 8, pp. 861-70, Aug 2005. 
[22] K. Alsop et al., "BRCA mutation frequency and patterns of treatment response in BRCA mutationpositive women with ovarian cancer: a report from the Australian Ovarian Cancer Study Group," (in eng), J Clin Oncol, vol. 30, no. 21, pp. 2654-63, Jul 2012.

[23] J. Boyd et al., "Clinicopathologic features of BRCA-linked and sporadic ovarian cancer," (in eng), JAMA, vol. 283, no. 17, pp. 2260-5, May 2000.

[24] K. L. Bolton et al., "Association between BRCA1 and BRCA2 mutations and survival in women with invasive epithelial ovarian cancer," (in eng), JAMA, vol. 307, no. 4, pp. 382-90, Jan 2012.

[25] E. Pujade-Lauraine et al., "Olaparib tablets as maintenance therapy in patients with platinumsensitive, relapsed ovarian cancer and a BRCA1/2 mutation (SOLO2/ENGOT-Ov21): a double-blind, randomised, placebo-controlled, phase 3 trial," (in eng), Lancet Oncol, Jul 2017.

[26] E. M. Swisher et al., "Rucaparib in relapsed, platinum-sensitive high-grade ovarian carcinoma (ARIEL2 Part 1): an international, multicentre, open-label, phase 2 trial," (in eng), Lancet Oncol, vol. 18, no. 1, pp. 75-87, Jan 2017.

[27] M. R. Mirza et al., "Niraparib Maintenance Therapy in Platinum-Sensitive, Recurrent Ovarian Cancer," (in eng), N Engl J Med, vol. 375, no. 22, pp. 2154-2164, 122016.

[28] C. G. A. R. Network, "Integrated genomic analyses of ovarian carcinoma," (in eng), Nature, vol. 474, no. 7353, pp. 609-15, Jun 2011.

[29] T. Pal et al., "BRCA1 and BRCA2 mutations account for a large proportion of ovarian carcinoma cases," (in eng), Cancer, vol. 104, no. 12, pp. 2807-16, Dec 2005.

[30] B. T. Hennessy et al., "Somatic mutations in BRCA1 and BRCA2 could expand the number of patients that benefit from poly (ADP ribose) polymerase inhibitors in ovarian cancer," (in eng), J Clin Oncol, vol. 28, no. 22, pp. 3570-6, Aug 2010.

[31] N. Turner, A. Tutt, and A. Ashworth, "Hallmarks of 'BRCAness' in sporadic cancers," (in eng), Nat Rev Cancer, vol. 4, no. 10, pp. 814-9, 102004.

[32] P. A. Konstantinopoulos et al., "Gene expression profile of BRCAness that correlates with responsiveness to chemotherapy and with outcome in patients with epithelial ovarian cancer," (in eng), J Clin Oncol, vol. 28, no. 22, pp. 3555-61, Aug 2010.

[33] S. Murata, C. Zhang, N. Finch, K. Zhang, L. Campo, and E. K. Breuer, "Predictors and Modulators of Synthetic Lethality: An Update on PARP Inhibitors and Personalized Medicine," (in eng), Biomed Res Int, vol. 2016, p. 2346585, 2016.

[34] J. Kang, A. D. D'Andrea, and D. Kozono, "A DNA repair pathway-focused score for prediction of outcomes in ovarian cancer treated with platinum-based chemotherapy," (in eng), J Natl Cancer Inst, vol. 104, no. 9, pp. 670-81, May 2012.

[35] K. P. Pennington et al., "Germline and somatic mutations in homologous recombination genes predict platinum response and survival in ovarian, fallopian tube, and peritoneal carcinomas," (in eng), Clin Cancer Res, vol. 20, no. 3, pp. 764-75, Feb 2014.

[36] N. Wagle et al., "High-throughput detection of actionable genomic alterations in clinical tumor samples by targeted, massively parallel sequencing," (in eng), Cancer Discov, vol. 2, no. 1, pp. 82-93, Jan 2012.

[37] J. A. Watkins, S. Irshad, A. Grigoriadis, and A. N. Tutt, "Genomic scars as biomarkers of homologous recombination deficiency and drug response in breast and ovarian cancers," (in eng), Breast Cancer Res, vol. 16, no. 3, p. 211, Jun 2014.

[38] L. B. Alexandrov et al., "Signatures of mutational processes in human cancer," (in eng), Nature, vol. 500, no. 7463, pp. 415-21, Aug 2013.

[39] R. A. Burrell et al., "Replication stress links structural and numerical cancer chromosomal instability," (in eng), Nature, vol. 494, no. 7438, pp. 492-496, Feb 2013.

[40] Y. K. Wang et al., "Genomic consequences of aberrant DNA repair mechanisms stratify ovarian cancer histotypes," (in eng), Nat Genet, vol. 49, no. 6, pp. 856-865, Jun 2017.

[41] A. Vanderstichele, P. Busschaert, S. Olbrecht, D. Lambrechts, and I. Vergote, "Genomic signatures as predictive biomarkers of homologous recombination deficiency in ovarian cancer," (in eng), Eur J Cancer, vol. 86, pp. 5-14, Nov 2017.

[42] C. K. Ng et al., "The role of tandem duplicator phenotype in tumour evolution in high-grade serous ovarian cancer," (in eng), J Pathol, vol. 226, no. 5, pp. 703-12, Apr 2012. 
[43] L. K. et al., "Quantification of genomic loss of heterozygosity enables prospective selection of ovarian cancer patients who may derive benefit from the PARP inhibitor rucaparib," vol. 51, ed. Eur J Cancer, 2015, pp. S531-532.

[44] R. L. Coleman et al., "Rucaparib maintenance treatment for recurrent ovarian carcinoma after response to platinum therapy (ARIEL3): a randomised, double-blind, placebo-controlled, phase 3 trial," (in eng), Lancet, vol. 390, no. 10106, pp. 1949-1961, Oct 2017.

[45] Mills et al., "Homologous recombination deficiency (HRD) score shows superior association with outcome compared to its individual score components (LOH, TAI, and LST scores) in platinumtreated serous ovarian cancer," vol. 141, ed. Gyn Oncol, 2016, pp. S2-3.

[46] H. Wei and X. Yu, "Functions of PARylation in DNA Damage Repair Pathways," (in eng), Genomics Proteomics Bioinformatics, vol. 14, no. 3, pp. 131-139, Jun 2016.

[47] A. Bürkle, "Physiology and pathophysiology of poly(ADP-ribosyl)ation," (in eng), Bioessays, vol. 23, no. 9, pp. 795-806, Sep 2001.

[48] J. M. de Murcia et al., "Requirement of poly(ADP-ribose) polymerase in recovery from DNA damage in mice and in cells," (in eng), Proc Natl Acad Sci U S A, vol. 94, no. 14, pp. 7303-7, Jul 1997.

[49] H. E. Bryant et al., "PARP is activated at stalled forks to mediate Mre11-dependent replication restart and recombination," (in eng), EMBO J, vol. 28, no. 17, pp. 2601-15, Sep 2009.

[50] H. E. Bryant et al., "Specific killing of BRCA2-deficient tumours with inhibitors of poly(ADP-ribose) polymerase," (in eng), Nature, vol. 434, no. 7035, pp. 913-7, Apr 2005.

[51] Y. Hu et al., "PARP1-driven poly-ADP-ribosylation regulates BRCA1 function in homologous recombination-mediated DNA repair," (in eng), Cancer Discov, vol. 4, no. 12, pp. 1430-47, Dec 2014.

[52] S. L. Rulten et al., "PARP-3 and APLF function together to accelerate nonhomologous end-joining," (in eng), Mol Cell, vol. 41, no. 1, pp. 33-45, Jan 2011.

[53] C. Boehler and F. Dantzer, "PARP-3, a DNA-dependent PARP with emerging roles in double-strand break repair and mitotic progression," (in eng), Cell Cycle, vol. 10, no. 7, pp. 1023-4, Apr 2011.

[54] A. Sonnenblick, E. de Azambuja, H. A. Azim, and M. Piccart, "An update on PARP inhibitors--moving to the adjuvant setting," (in eng), Nat Rev Clin Oncol, vol. 12, no. 1, pp. 27-41, Jan 2015.

[55] B. A. Gibson and W. L. Kraus, "New insights into the molecular and cellular functions of poly(ADPribose) and PARPs," (in eng), Nat Rev Mol Cell Biol, vol. 13, no. 7, pp. 411-24, Jun 2012.

[56] P. Jagtap and C. Szabó, "Poly(ADP-ribose) polymerase and the therapeutic effects of its inhibitors," (in eng), Nat Rev Drug Discov, vol. 4, no. 5, pp. 421-40, May 2005.

[57] H. Farmer et al., "Targeting the DNA repair defect in BRCA mutant cells as a therapeutic strategy," (in eng), Nature, vol. 434, no. 7035, pp. 917-21, Apr 2005.

[58] C. L. Scott, E. M. Swisher, and S. H. Kaufmann, "Poly (ADP-ribose) polymerase inhibitors: recent advances and future development," (in eng), J Clin Oncol, vol. 33, no. 12, pp. 1397-406, Apr 2015.

[59] A. Ashworth, "A synthetic lethal therapeutic approach: poly(ADP) ribose polymerase inhibitors for the treatment of cancers deficient in DNA double-strand break repair," (in eng), J Clin Oncol, vol. 26, no. 22, pp. 3785-90, Aug 2008.

[60] A. G. Patel, J. N. Sarkaria, and S. H. Kaufmann, "Nonhomologous end joining drives poly(ADP-ribose) polymerase (PARP) inhibitor lethality in homologous recombination-deficient cells," (in eng), Proc Natl Acad Sci U S A, vol. 108, no. 8, pp. 3406-11, Feb 2011.

[61] B. Wang, D. Chu, Y. Feng, Y. Shen, M. Aoyagi-Scharber, and L. E. Post, "Discovery and Characterization of (8S,9R)-5-Fluoro-8-(4-fluorophenyl)-9-(1-methyl-1H-1,2,4-triazol-5-yl)-2,7,8,9tetrahydro-3H-pyrido[4,3,2-de]phthalazin-3-one (BMN 673, Talazoparib), a Novel, Highly Potent, and Orally Efficacious Poly(ADP-ribose) Polymerase-1/2 Inhibitor, as an Anticancer Agent," (in eng), J Med Chem, vol. 59, no. 1, pp. 335-57, Jan 2016.

[62] J. Murai et al., "Trapping of PARP1 and PARP2 by Clinical PARP Inhibitors," (in eng), Cancer Res, vol. 72, no. 21, pp. 5588-99, Nov 2012.

[63] J. Murai et al., "Stereospecific PARP trapping by BMN 673 and comparison with olaparib and rucaparib," (in eng), Mol Cancer Ther, vol. 13, no. 2, pp. 433-43, Feb 2014.

[64] J. K. Horton, D. F. Stefanick, R. Prasad, N. R. Gassman, P. S. Kedar, and S. H. Wilson, "Base excision repair defects invoke hypersensitivity to PARP inhibition," (in eng), Mol Cancer Res, vol. 12, no. 8, pp. 1128-39, Aug 2014. 
Lymparza [package insert], Astra Zeneca 2014, https://www.accessdata.fda.gov/drugsatfda_docs/label/2014/206162lbl.pdf

$[66$

Lymparza [package insert], Astra

Zeneca

2017, https://www.accessdata.fda.gov/drugsatfda_docs/label/2017/208558s000lbl.pdf.

[67] G. Kim et al., "FDA Approval Summary: Olaparib Monotherapy in Patients with Deleterious Germline BRCA-Mutated Advanced Ovarian Cancer Treated with Three or More Lines of Chemotherapy," (in eng), Clin Cancer Res, vol. 21, no. 19, pp. 4257-61, Oct 2015.

[68] P. C. Fong et al., "Inhibition of poly(ADP-ribose) polymerase in tumors from BRCA mutation carriers," (in eng), N Engl J Med, vol. 361, no. 2, pp. 123-34, Jul 2009.

[69] P. C. Fong et al., "Poly(ADP)-ribose polymerase inhibition: frequent durable responses in BRCA carrier ovarian cancer correlating with platinum-free interval," (in eng), J Clin Oncol, vol. 28, no. 15, pp. 2512-9, May 2010.

[70] M. W. Audeh et al., "Oral poly(ADP-ribose) polymerase inhibitor olaparib in patients with BRCA1 or BRCA2 mutations and recurrent ovarian cancer: a proof-of-concept trial," (in eng), Lancet, vol. 376, no. 9737, pp. 245-51, Jul 2010.

[71] S. B. Kaye et al., "Phase II, open-label, randomized, multicenter study comparing the efficacy and safety of olaparib, a poly (ADP-ribose) polymerase inhibitor, and pegylated liposomal doxorubicin in patients with BRCA1 or BRCA2 mutations and recurrent ovarian cancer," (in eng), J Clin Oncol, vol. 30, no. 4, pp. 372-9, Feb 2012.

[72] K. A. Gelmon et al., "Olaparib in patients with recurrent high-grade serous or poorly differentiated ovarian carcinoma or triple-negative breast cancer: a phase 2, multicentre, open-label, nonrandomised study," (in eng), Lancet Oncol, vol. 12, no. 9, pp. 852-61, Sep 2011.

[73] J. Ledermann et al., "Olaparib maintenance therapy in platinum-sensitive relapsed ovarian cancer," (in eng), N Engl J Med, vol. 366, no. 15, pp. 1382-92, Apr 2012.

[74] J. A. Ledermann, "PARP inhibitors in ovarian cancer," vol. Supplement 1, ed. Annals of Oncology, 2016.

[75] J. Ledermann et al., "Olaparib maintenance therapy in patients with platinum-sensitive relapsed serous ovarian cancer: a preplanned retrospective analysis of outcomes by BRCA status in a randomised phase 2 trial," (in eng), Lancet Oncol, vol. 15, no. 8, pp. 852-61, Jul 2014.

[76] J. A. Ledermann et al., "Overall survival in patients with platinum-sensitive recurrent serous ovarian cancer receiving olaparib maintenance monotherapy: an updated analysis from a randomised, placebo-controlled, double-blind, phase 2 trial," (in eng), Lancet Oncol, vol. 17, no. 11, pp. 15791589, Nov 2016.

[77] A. M. Oza et al., "Olaparib combined with chemotherapy for recurrent platinum-sensitive ovarian cancer: a randomised phase 2 trial," (in eng), Lancet Oncol, vol. 16, no. 1, pp. 87-97, Jan 2015.

[78] Penson $\mathrm{R}$ et al., "Efficacy of olaparib maintenance therapy in patients with platinum-sensitive relapsed ovarian cancer by number of lines of prior chemotherapy: Phase III SOLO2 trial (ENGOT Ov-21)," vol. 28, ed. Annals of Oncology 2017, pp. v330-v354.

[79] Friedlander $\mathrm{M}$ et al., "Health-related quality of life (HRQOL) and patient-centered outcomes with maintenance olaparib compared with placebo following chemotherapy in patients with germline (g) BRCA-mutated $(\mathrm{m})$ platinum-sensitive relapsed serous ovarian cancer (PSR SOC): SOLO2 phase III trial.," vol. 35, ed. J Clin Oncol, 2017.

[80] B. Kaufman et al., "Olaparib monotherapy in patients with advanced cancer and a germline BRCA1/2 mutation," (in eng), J Clin Oncol, vol. 33, no. 3, pp. 244-50, Jan 2015.

[81] S. M. Domchek et al., "Efficacy and safety of olaparib monotherapy in germline BRCA1/2 mutation carriers with advanced ovarian cancer and three or more lines of prior therapy," (in eng), Gynecol Oncol, vol. 140, no. 2, pp. 199-203, Feb 2016.

[82] U. A. Matulonis et al., "Olaparib monotherapy in patients with advanced relapsed ovarian cancer and a germline BRCA1/2 mutation: a multistudy analysis of response rates and safety," (in eng), Ann Oncol, vol. 27, no. 6, pp. 1013-9, Jun 2016.

[83] J. Mateo et al., "An Adaptive Study to Determine the Optimal Dose of the Tablet Formulation of the PARP Inhibitor Olaparib," (in eng), Target Oncol, vol. 11, no. 3, pp. 401-15, Jun 2016. 
[84] J. F. Liu et al., "Combination cediranib and olaparib versus olaparib alone for women with recurrent platinum-sensitive ovarian cancer: a randomised phase 2 study," (in eng), Lancet Oncol, vol. 15, no. 11, pp. 1207-14, Oct 2014.

[85] M. K. McConechy et al., "Use of mutation profiles to refine the classification of endometrial carcinomas," (in eng), J Pathol, vol. 228, no. 1, pp. 20-30, Sep 2012.

[86] Zejula [package insert]. Waltham, MA: Tesaro Inc;2017 https://www.accessdata.fda.gov/drugsatfda_docs/label/2017/208447/bl.pdf.

[87] S. K. Sandhu et al., "The poly(ADP-ribose) polymerase inhibitor niraparib (MK4827) in BRCA mutation carriers and patients with sporadic cancer: a phase 1 dose-escalation trial," (in eng), Lancet Oncol, vol. 14, no. 9, pp. 882-92, Aug 2013.

[88] Matulonis UA et al., " Long-term benefit of niraparib treatment of recurrent ovarian cancer (OC)," vol. 35, ed. J Clin Oncol, 2017.

[89] Mirza MR et al., "Efficacy of niraparib on progression-free survival (PFS) in patients (pts) with recurrent ovarian cancer (OC) with partial response (PR) to the last platinum-based chemotherapy," vol. 35, ed. J Clin Oncol, 2017.

[90] Del Campo J et al., "The successful phase 3 niraparib ENGOT-OV16/NOVA trial included a substantial number of patients with platinum resistant ovarian cancer (OC)," vol. 35, ed. J Clin Oncol, 2017.

[91] M. M, "how to use Niraparib in reccurrent ovarian cancer and the impact on patient quality of life," ed. European Society of Gynecology Oncology (ESGO) Congress, November 2017, 2017.

[92] Rubraca [package Clovis Oncology 2016, https://www.accessdata.fda.gov/drugsatfda_docs/label/2016/209115s000lbl.pdf.

[93] Kristeleit RS et al., "Phase 1/2 study of oral rucaparib: final phase 1 results.," vol. 32, ed. J Clin Oncol, 2014.

[94] http://clovisoncology.com/files/rubraca-prescribing-info.pdf.

[95] R. Kristeleit et al., "A Phase I-II Study of the Oral PARP Inhibitor Rucaparib in Patients with Germline BRCA1/2-Mutated Ovarian Carcinoma or Other Solid Tumors," (in eng), Clin Cancer Res, vol. 23, no. 15, pp. 4095-4106, Aug 2017.

[96] Y. Drew et al., "Phase 2 multicentre trial investigating intermittent and continuous dosing schedules of the poly(ADP-ribose) polymerase inhibitor rucaparib in germline BRCA mutation carriers with advanced ovarian and breast cancer," (in eng), Br J Cancer, vol. 114, no. 12, p. e21, Jun 2016.

[98] De Bono JS et al., "First-in-human trial of novel oral PARP inhibitor BMN 673 in patients with solid tumors," vol. 31, ed. J Clin Oncol, 2013.

[99] J. de Bono et al., "Phase I, Dose-Escalation, Two-Part Trial of the PARP Inhibitor Talazoparib in Patients with Advanced Germline BRCA1/2 Mutations and Selected Sporadic Cancers," (in eng), Cancer Discov, vol. 7, no. 6, pp. 620-629, 062017.

[100] M. S. Dhawan et al., "Differential Toxicity in Patients with and without DNA Repair Mutations: Phase I Study of Carboplatin and Talazoparib in Advanced Solid Tumors," (in eng), Clin Cancer Res, vol. 23, no. 21, pp. 6400-6410, Nov 2017.

[101] Turner NC et al., "Final results of a phase 2 study of talazoparib (TALA) following platinum or multiple cytotoxic regimens in advanced breast cancer patients (pts) with germline BRCA1/2 mutations (ABRAZO)." vol. 35, ed. J Clin Oncol, 2017.

[102] S. Kummar et al., "Phase 0 clinical trial of the poly (ADP-ribose) polymerase inhibitor ABT-888 in patients with advanced malignancies," (in eng), J Clin Oncol, vol. 27, no. 16, pp. 2705-11, Jun 2009.

[103] Puhalla $S$ et al., "Final results of a phase 1 study of single-agent veliparib (V) in patients (pts) with either BRCA1/2-mutated

cancer (BRCA+), platinum-refractory ovarian, or basal-like breast cancer (BRCA-wt)," vol. 32, ed. J Clin Oncol, 2014. 
[104] R. L. Coleman et al., "A phase II evaluation of the potent, highly selective PARP inhibitor veliparib in the treatment of persistent or recurrent epithelial ovarian, fallopian tube, or primary peritoneal cancer in patients who carry a germline BRCA1 or BRCA2 mutation - An NRG Oncology/Gynecologic Oncology Group study," (in eng), Gynecol Oncol, vol. 137, no. 3, pp. 386-91, Jun 2015.

[105] K. D. Steffensen, P. Adimi, and A. Jakobsen, "Veliparib Monotherapy to Patients With BRCA Germ Line Mutation and Platinum-Resistant or Partially Platinum-Sensitive Relapse of Epithelial Ovarian Cancer: A Phase I/II Study," (in eng), Int J Gynecol Cancer, vol. 27, no. 9, pp. 1842-1849, Nov 2017.

[106] S. Nishio et al., "Phase 1 study of veliparib with carboplatin and weekly paclitaxel in Japanese patients with newly diagnosed ovarian cancer," (in eng), Cancer Sci, vol. 108, no. 11, pp. 2213-2220, Nov 2017.

[107] L. M. Landrum et al., "A phase I trial of pegylated liposomal doxorubicin (PLD), carboplatin, bevacizumab and veliparib in recurrent, platinum-sensitive ovarian, primary peritoneal, and fallopian tube cancer: An NRG Oncology/Gynecologic Oncology Group study," (in eng), Gynecol Oncol, vol. 140, no. 2, pp. 204-9, Feb 2016.

[108] S. Kummar et al., "Randomized Trial of Oral Cyclophosphamide and Veliparib in High-Grade Serous Ovarian, Primary Peritoneal, or Fallopian Tube Cancers, or BRCA-Mutant Ovarian Cancer," (in eng), Clin Cancer Res, vol. 21, no. 7, pp. 1574-82, Apr 2015.

[109] K. A. Reiss et al., "A Phase I study of veliparib (ABT-888) in combination with low-dose fractionated whole abdominal radiation therapy in patients with advanced solid malignancies and peritoneal carcinomatosis," (in eng), Clin Cancer Res, vol. 21, no. 1, pp. 68-76, Jan 2015.

[110] S. Pignata, S. C. Cecere, A. Du Bois, P. Harter, and F. Heitz, "Treatment of recurrent ovarian cancer," ed. Annals of Oncology, Volume 28, Issue suppl_8, 12017 , pp. viii51-viii56.

[111] C. Aghajanian, B. Goff, L. R. Nycum, Y. V. Wang, A. Husain, and S. V. Blank, "Final overall survival and safety analysis of OCEANS, a phase 3 trial of chemotherapy with or without bevacizumab in patients with platinum-sensitive recurrent ovarian cancer," (in eng), Gynecol Oncol, vol. 139, no. 1, pp. 10-6, Oct 2015.

[112] A. Ohmoto and S. Yachida, "Current status of poly(ADP-ribose) polymerase inhibitors and future directions," (in eng), Onco Targets Ther, vol. 10, pp. 5195-5208, 2017.

[113] B. G. Bitler, Z. L. Watson, L. J. Wheeler, and K. Behbakht, "PARP inhibitors: Clinical utility and possibilities of overcoming resistance," (in eng), Gynecol Oncol, vol. 147, no. 3, pp. 695-704, 12 2017.

[114] Oza AM et al., "Quality of life in patients with recurrent ovarian cancer treated with niraparib: results from ENGOT-OV16/NOVA trial," vol. 28 ed. Annals of Oncology 2017.

[115] N. McCabe et al., "Deficiency in the repair of DNA damage by homologous recombination and sensitivity to poly(ADP-ribose) polymerase inhibition," (in eng), Cancer Res, vol. 66, no. 16, pp. 8109-15, Aug 2006.

[116] D. Durocher, A. Orthwein, and S. Noordermeer, "Homologous recombination factors. WO2016138574A1.," 2016.

[117] D. Dietrich, "Method for determining a mutation in genomic dna, use of the method and kit for carrying out said method. WO2017008912A1.," 2017.

[118] V. Abkevich, A. Gutin, K. Timms, and J. Lanchbury, "Methods and materials for assessing loss of heterozygosity. 9388472," 2016.

[119] A. Cerrato, F. Morra, and A. Celetti, "Use of poly ADP-ribose polymerase [PARP] inhibitors in cancer cells bearing DDR defects: the rationale for their inclusion in the clinic," (in eng), J Exp Clin Cancer Res, vol. 35, no. 1, p. 179, Nov 2016.

[120] G. von Minckwitz et al., "Cytoplasmic poly(adenosine diphosphate-ribose) polymerase expression is predictive and prognostic in patients with breast cancer treated with neoadjuvant chemotherapy," (in eng), J Clin Oncol, vol. 29, no. 16, pp. 2150-7, Jun 2011.

[121] A. J. Wiggans, G. K. Cass, A. Bryant, T. A. Lawrie, and J. Morrison, "Poly(ADP-ribose) polymerase (PARP) inhibitors for the treatment of ovarian cancer," (in eng), Cochrane Database Syst Rev, no. 5, p. CD007929, May 2015.

[122] A. Montoni, M. Robu, E. Pouliot, and G. M. Shah, "Resistance to PARP-Inhibitors in Cancer Therapy," (in eng), Front Pharmacol, vol. 4, p. 18, 2013. 
[123] B. Norquist et al., "Secondary somatic mutations restoring BRCA1/2 predict chemotherapy resistance in hereditary ovarian carcinomas," (in eng), J Clin Oncol, vol. 29, no. 22, pp. 3008-15, Aug 2011.

[124] K. K. Dhillon, E. M. Swisher, and T. Taniguchi, "Secondary mutations of BRCA1/2 and drug resistance," (in eng), Cancer Sci, vol. 102, no. 4, pp. 663-9, Apr 2011.

[125] Y. Yun, "Treatment of BRCA1-defective cancer or resistant cancers. US20170035737," 2017.

[126] O. Kondrashova et al., "Secondary Somatic Mutations Restoring RAD51C and RAD51D Associated with Acquired Resistance to the PARP Inhibitor Rucaparib in High-Grade Ovarian Carcinoma," (in eng), Cancer Discov, vol. 7, no. 9, pp. 984-998, 092017.

[127] S. Rottenberg et al., "High sensitivity of BRCA1-deficient mammary tumors to the PARP inhibitor AZD2281 alone and in combination with platinum drugs," (in eng), Proc Natl Acad Sci U S A, vol. 105, no. 44, pp. 17079-84, Nov 2008.

[128] A. M. Patch et al., "Whole-genome characterization of chemoresistant ovarian cancer," (in eng), Nature, vol. 521, no. 7553, pp. 489-94, May 2015.

[129] !!! INVALID CITATION !!!

[130] S. F. Blanchette, D. C. Drummond, J. B. Fitzgerald, and V. Moyo, "Combination therapy for cancer treatment. US20170049767," 2017.

[131] D. Proia, "Combination therapy for treatment of ovarian cancer. WO2017151554A1.," 2017.

[132] S. F. Blanchette, D. C. Drummond, J. B. Fitzgerald, and V. Moyo, "Combination therapy using liposomal irinotecan and a PARP inhibitor for cancer treatment. WO2017031442A1," 2017.

[133] S. N. Seyedin et al., "Strategies for combining immunotherapy with radiation for anticancer therapy," (in eng), Immunotherapy, vol. 7, no. 9, pp. 967-980, 2015.

[134] E. C. Fields, W. P. McGuire, L. Lin, and S. M. Temkin, "Radiation Treatment in Women with Ovarian Cancer: Past, Present, and Future," (in eng), Front Oncol, vol. 7, p. 177, 2017.

[135] J. Hamanishi et al., "Safety and Antitumor Activity of Anti-PD-1 Antibody, Nivolumab, in Patients With Platinum-Resistant Ovarian Cancer," (in eng), J Clin Oncol, vol. 33, no. 34, pp. 4015-22, Dec 2015.

[136] W. K. Holloman, "Unraveling the mechanism of BRCA2 in homologous recombination," (in eng), Nat Struct Mol Biol, vol. 18, no. 7, pp. 748-54, Jul 2011.

[137] G. Mittica, S. Genta, M. Aglietta, and G. Valabrega, "Immune Checkpoint Inhibitors: A New Opportunity in the Treatment of Ovarian Cancer?," (in eng), Int J Mol Sci, vol. 17, no. 7, Jul 2016.

[138] D. Lambrechts, "Novel markers for detecting microsatellite instability in cancer and determining synthetic lethality with inhibition of the DNA base excision repair pathway. WO2013153130A1.," 2013.

[139] R. E. Reinbolt and J. L. Hays, "The Role of PARP Inhibitors in the Treatment of Gynecologic Malignancies," (in eng), Front Oncol, vol. 3, p. 237, Oct 2013.

[140] T. W. Kim, D. H. Jin, S. W. Hong, J. S. Shin, I. Y. Hwang, and J. H. Kim, "Novel biomarker for predicting sensitivity to PARP inhibitor, and use thereof. WO2016018089. ," 2016.

[141] K. Yamamoto and M. Okazaki, "Polymeric Compound Having Camptothecin Compound And AntiCancer Effect Enhancer Bound Thereto, And Use of Same. US20150290185 (EP2918292A1)," 2015.

[142] D. J. Slamon, R. S. Finn, and D. Conklin, "Biomarkers of Response to Inhibition of Poly- ADP Ribose Polymerase (PARP) in Cancer. US20160138114 (WO2014205105A1).", 2014.

[143] JP2017504623A, "Use of PARP inhibitor for treating the patient of a breast cancer or an ovarian cancer who shows loss of heterozygosis. JP2017504623A (WO2015108986A1)."

[144] D. Cox, A. Kremer, S. McGonigle, and J. Wu, "Use of eribulin and poly (adp ribose) polymerase (parp) inhibitors as combination therapy for the treatment of cancer. US20170100368 (WO 2015184145A1)," 2017.

[145] N. Johnson, "Methods for determining PARP inhibitor and platinum resistance in cancer therapy. US20150344968," 2015.

[146] S. Kadiyala et al., "Platinum compounds, compositions, and uses thereof. WO2016209935A1," 2016. 
[147] D. M. Goldenberg and T. M. Cardillo, "Combining anti-HLA- DR or anti-Trop- 2 antibodies with microtubule inhibitors, PARP inhibitors, bruton kinase inhibitors or phosphoinositide 3-kinase inhibitor significantly improves therapeutic outcome in cancer. 9707302 (US20170274093)," 2017.

[148] M. Dutreix, "Use of a combination of dbait molecule and PARP inhibitors to treat cancer. WO2017013237A1.," 2017.

[149] S. J. Shuttleworth and A. D. Whale, "Compositions comprising phosphoinositide 3- kinase inhibitors and a second antiproliferative agent .WO 2017029517A1.," 2017.

Figure 1. Principal mechanisms of action of PARPIs

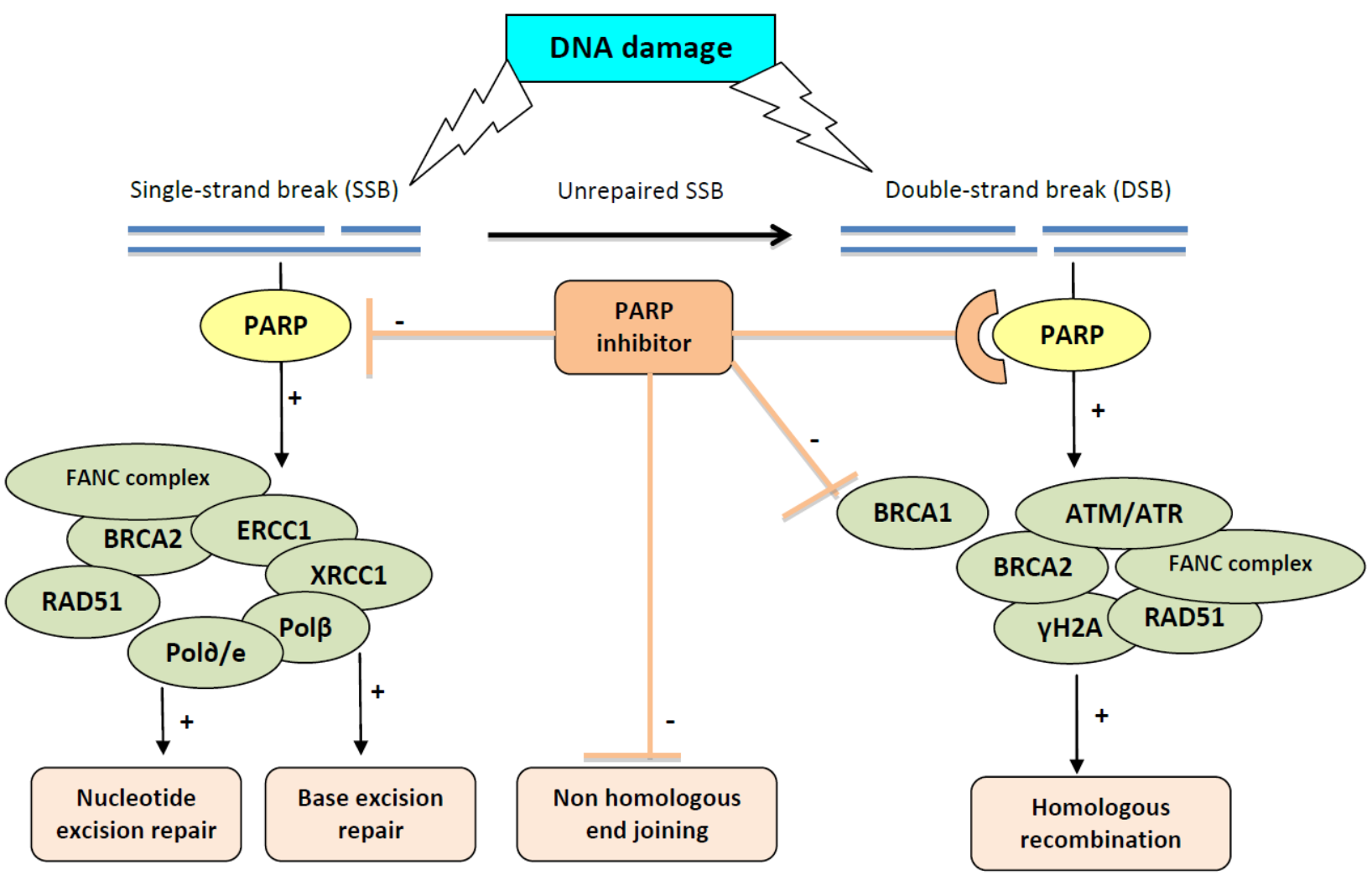


Figure 2. Olaparib chemical structure

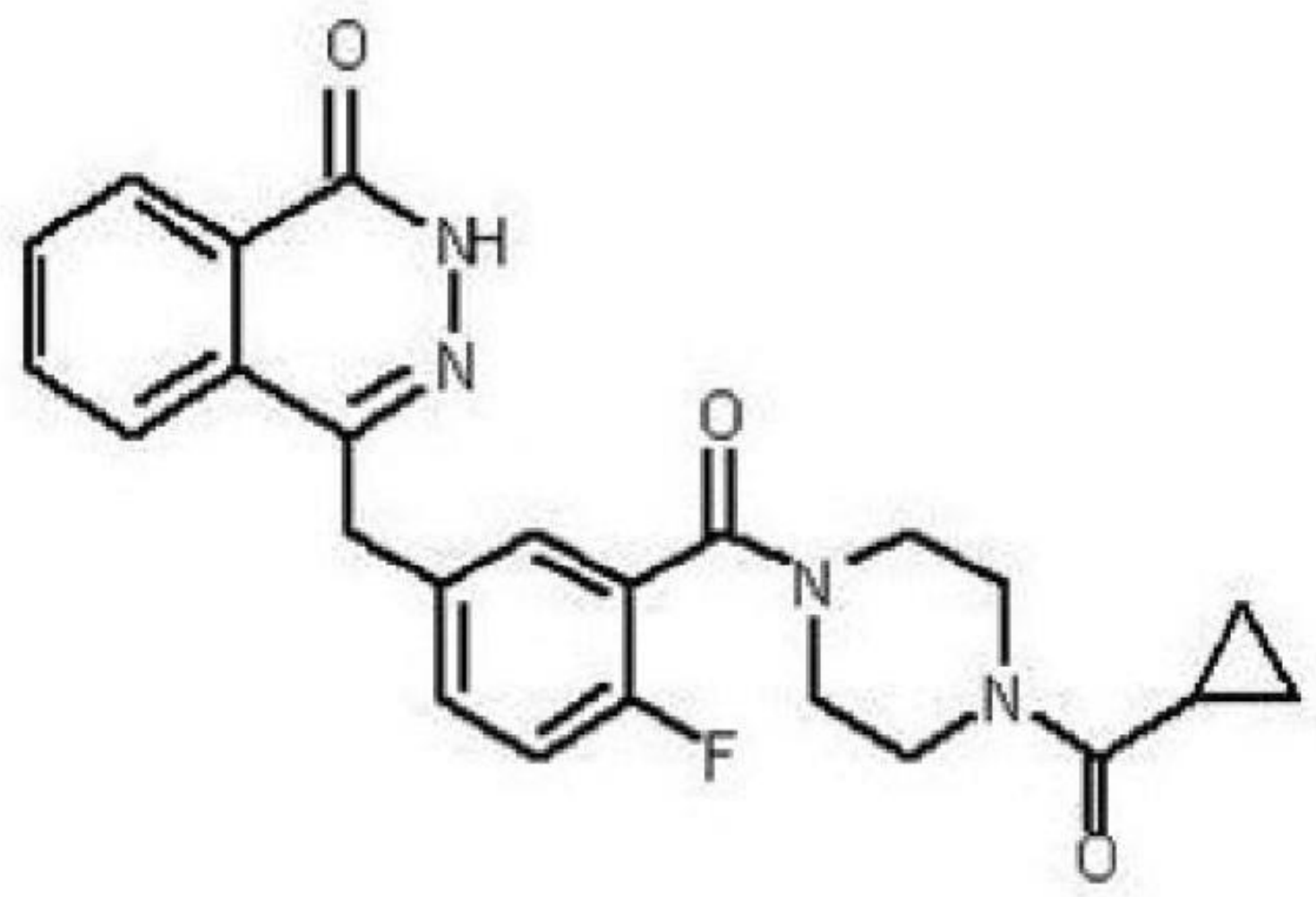


Figure 3. Niraparib chemical structure
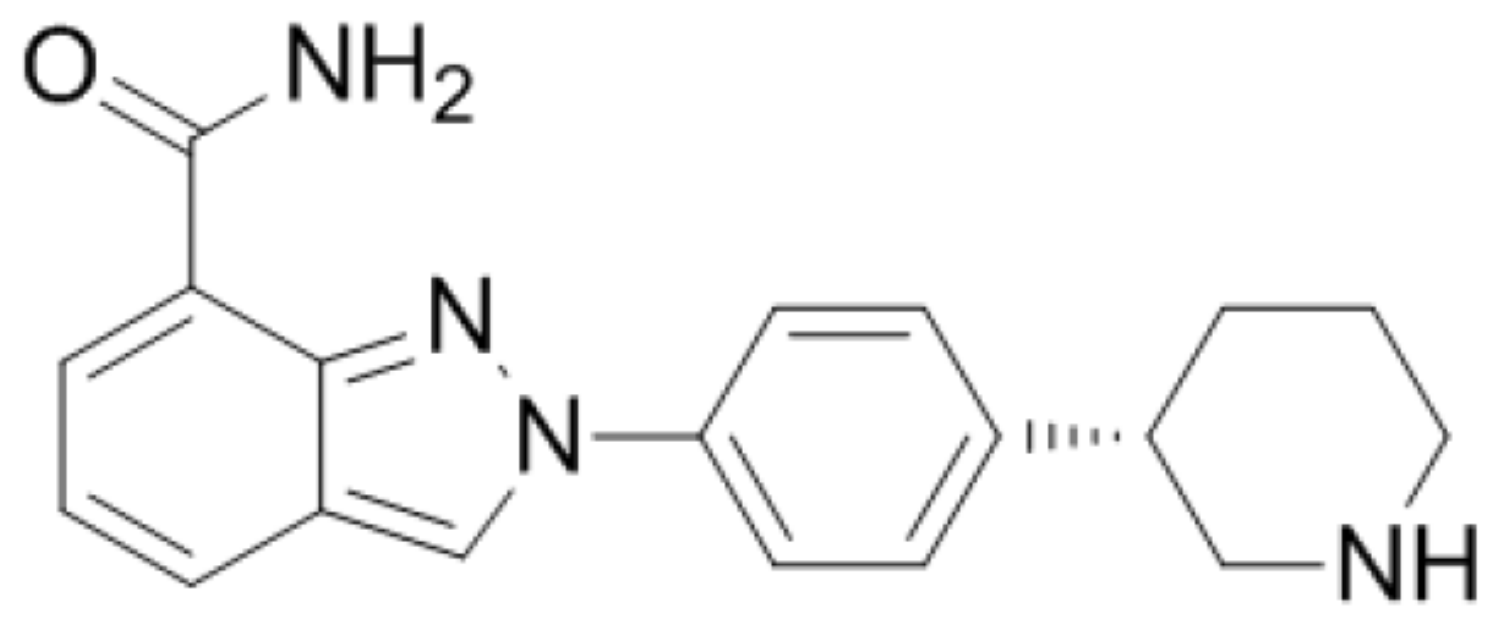
Figure 4. Rucaparib chemical structure

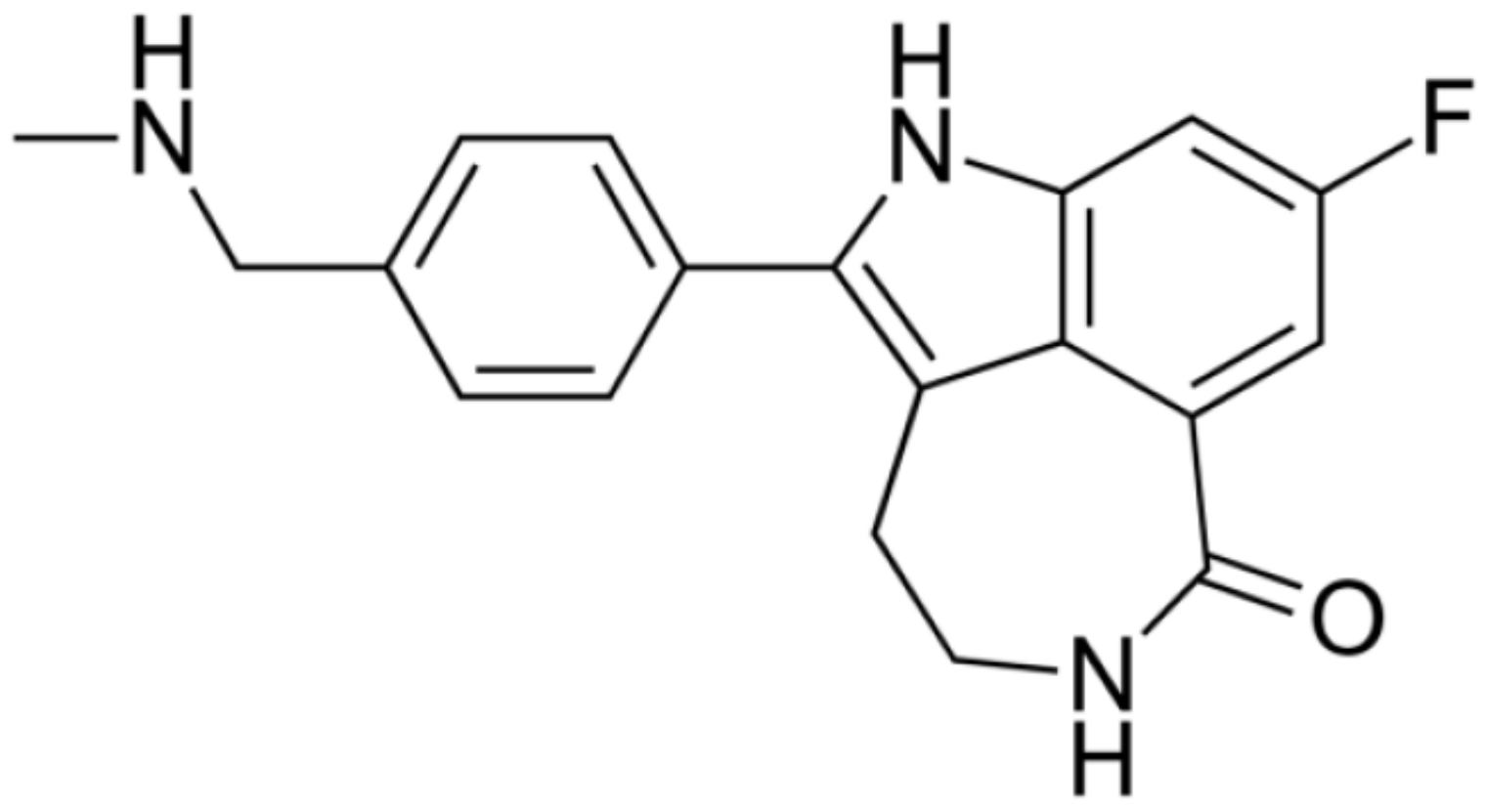


Figure 5. Talazoparib chemical structure

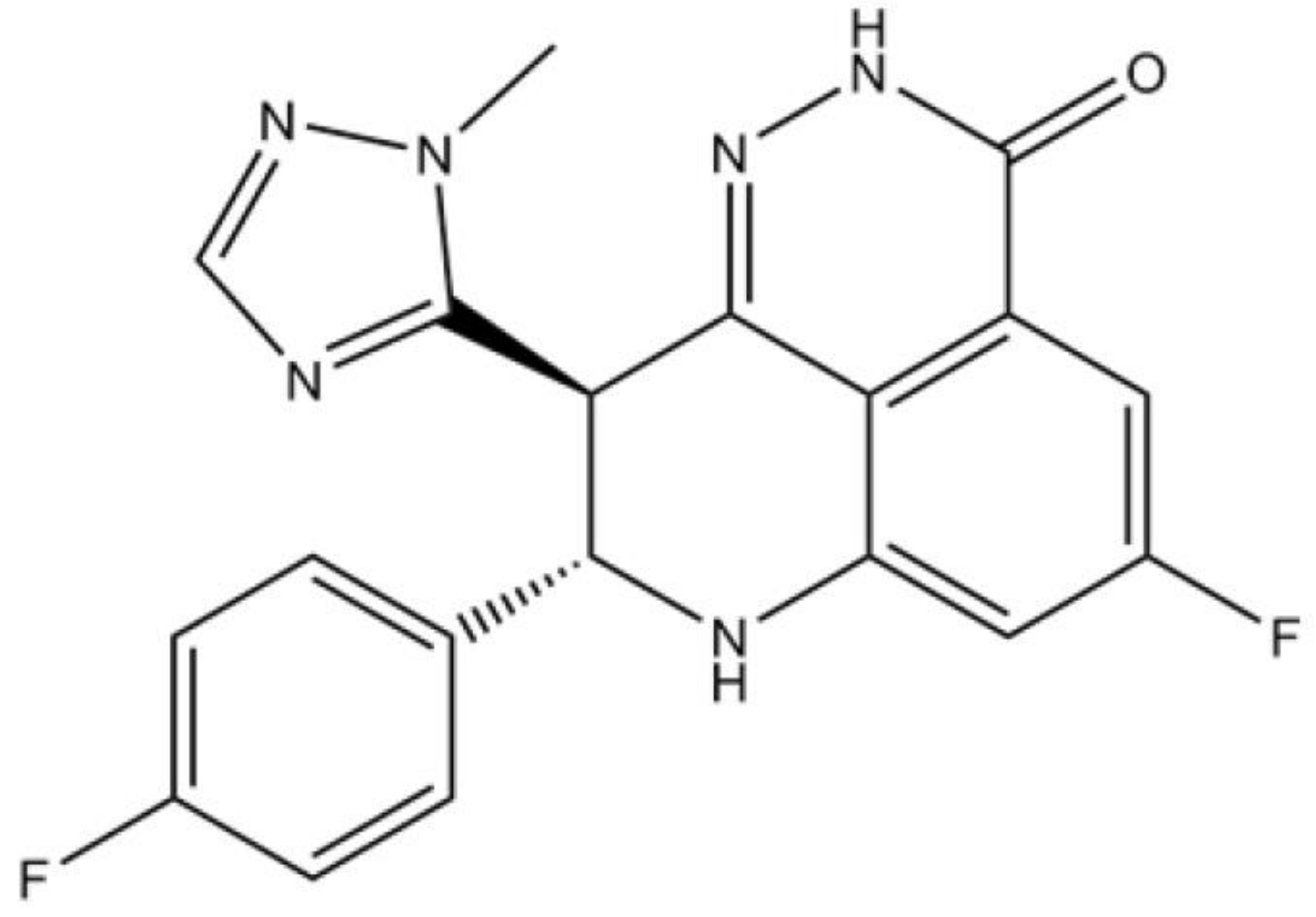


Figure 6. Veliparib chemical structure

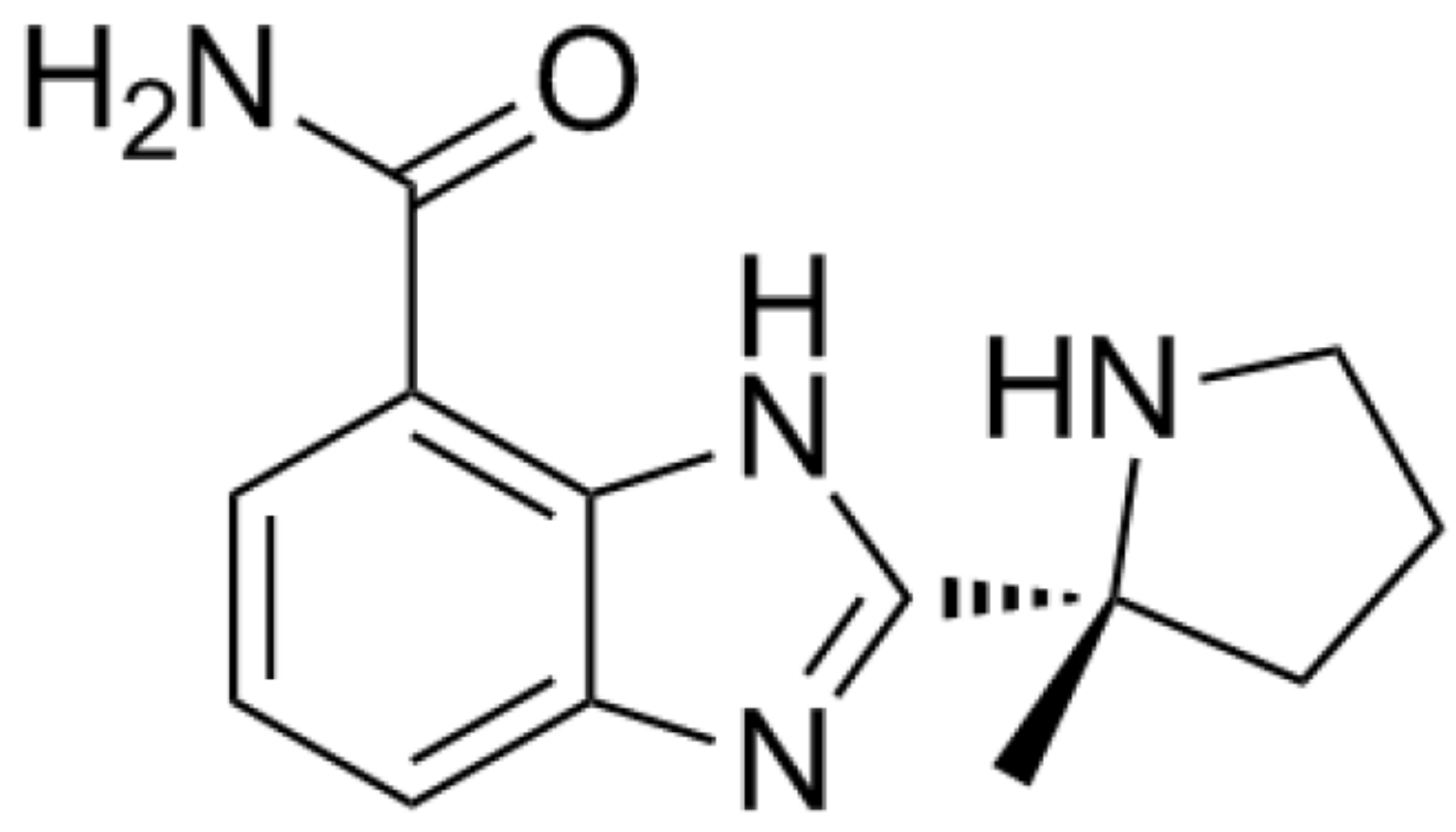




\begin{tabular}{|c|c|c|c|c|c|}
\hline & Setting & Line of therapy & Phase & Status & Trial identifier \\
\hline Olaparib & $\begin{array}{c}\text { Newly diagnosed } \\
\text { BRCAm }\end{array}$ & $1 \mathrm{~L}$ & 3 & Not yet recruiting & NCT01844986 \\
\hline Olaparib & Neoadjuvant & $1 \mathrm{~L}$ & 2 & Recruiting & NCT02489006 \\
\hline $\begin{array}{l}\text { Olaparib+Bevacizum } \\
\text { ab }\end{array}$ & gBRCAm manteinance & $1 \mathrm{~L}$ & 3 & Recruiting & NCT02477644 \\
\hline $\begin{array}{l}\text { Olaparib versus } \\
\text { single agent } \\
\text { chemotherapy }\end{array}$ & $\begin{array}{c}\text { Platinum-sensitive } \\
\text { gBRCAm }\end{array}$ & $\geq 3$ & 3 & Recruiting & NCT02282020 \\
\hline $\begin{array}{l}\text { Olaparib versus } \\
\text { chemotherapy }\end{array}$ & $\begin{array}{c}\text { Platinum-sensitive and } \\
\text { resistant } \\
\text { regardless BRCA } \\
\text { status }\end{array}$ & $\geq 2$ & 2 & Recruiting & NCT02822157 \\
\hline Olaparib+Cediranib & $\begin{array}{c}\text { Relapsed after Olaparib } \\
\text { treatment }\end{array}$ & $\geq 2$ & 2 & Not yet recruiting & NCT02340611 \\
\hline Olaparib & $\begin{array}{l}\text { Platinum-sensitive } \\
\text { HRD positive }\end{array}$ & $\geq 2$ & 2 & Recruiting & NCT02983799 \\
\hline Olaparib & $\begin{array}{c}\text { EOC pretreated with } \\
\text { Olaparib } \\
\text { responding to } \\
\text { platinum } \\
\text { compound }\end{array}$ & $\geq 2$ & 3 & Recruiting & NCT03106987 \\
\hline Olaparib+Cediranib & $\begin{array}{c}\text { Platinum-sensitive and } \\
\text { resistant relapsed } \\
\text { after PARPi }\end{array}$ & $\geq 2$ & 2 & Recruiting & NCT02681237 \\
\hline $\begin{array}{l}\text { Olaparib+Cediranib } \\
\text { after chemotherapy }\end{array}$ & $\begin{array}{c}\text { Platinum-sensitive } \\
\text { relapsed BRCAm } \\
\text { pretreated with } \\
\text { Olaparib }\end{array}$ & $\geq 2$ & 1 & Recruiting & NCT02855697 \\
\hline Olaparib+Cediranib & Platinum-sensitive & $\geq 2$ & 3 & Recruiting & NCT02446600 \\
\hline
\end{tabular}




\begin{tabular}{|c|c|c|c|c|c|}
\hline & BRCAm & & & & \\
\hline Olaparib+Cediranib & $\begin{array}{c}\text { Platinum resistant } \\
\text { BRCA WT }\end{array}$ & $\geq 3$ & 2 & Recruiting & NCT02889900 \\
\hline $\begin{array}{l}\text { Olaparib+Cediranib } \\
\text { versus Paclitaxel }\end{array}$ & $\begin{array}{l}\text { Platinum } \\
\text { resistant/refractor } \\
\mathrm{y}\end{array}$ & $\geq 2$ & 2 & Recruiting & NCT03314740 \\
\hline $\begin{array}{l}\text { Olaparib } \pm \text { Cediranib } \\
\text { versus Paclitaxel }\end{array}$ & $\begin{array}{c}\text { Platinum resistant } \\
\text { BRCAm }\end{array}$ & $\geq 2$ & 2 & Recruiting & NCT03117933 \\
\hline Olaparib \pm Cediranib & $\begin{array}{l}\text { Maintenance after } \\
\text { platinum response }\end{array}$ & 2 & 3 & Not yet Recruiting & NCT03278717 \\
\hline $\begin{array}{l}\text { Olaparib and/or } \\
\text { cediranib+anti-PD1 }\end{array}$ & $\begin{array}{c}\text { Platinum-sensitive or } \\
\text { resistant }\end{array}$ & $\geq 3$ & $1 / 2$ & Recruiting & NCT02484404 \\
\hline $\begin{array}{l}\text { Olaparib+tremelimu } \\
\text { mab }\end{array}$ & $\begin{array}{l}\text { Platinum-sensitive or } \\
\text { resistant BRCAm }\end{array}$ & $\geq 2$ & $1 / 2$ & Recruiting & NCT02571725 \\
\hline $\begin{array}{l}\text { Olaparib+Tremelim } \\
\text { umab+Durvalumab }\end{array}$ & $\begin{array}{l}\text { Platinum-sensitive or } \\
\text { resistant BRCAm }\end{array}$ & $\leq 3$ & $1 / 2$ & Recruiting & NCT02953457 \\
\hline Olaparib+PLD & Platinum resistant & $\geq 2$ & 2 & Not yet recruiting & NCT03161132 \\
\hline Olaparib+PM01183 & Platinum resistant & $\geq 2$ & $1 / 2$ & Recruiting & NCT02684318 \\
\hline Olaparib & $\begin{array}{l}\text { Maintenance } \\
\text { BRCAm/HRD+ }\end{array}$ & 2 & 4 & Recruiting & NCT02476968 \\
\hline Olaparib+Onalespib & Platinum resistant & $\geq 3$ & 1 & Recruiting & NCT02898207 \\
\hline Olaparib+AZD1775 & Platinum resistant & $\geq 3$ & $1 b$ & Recruiting & NCT02511975 \\
\hline
\end{tabular}


Table 2. Ongoing clinical trials involving Niraparib in EOC

\begin{tabular}{lccccl}
\hline \multicolumn{1}{c}{ Combination } & \multicolumn{1}{c}{ Setting } & Line of therapy & Phase & Status & Trial identifier \\
\hline Niraparib & Platinum-sensitive & $1 \mathrm{~L}$ & 3 & recruiting & NCT02655016 \\
Niraparib & Platinum-resistant & $>3 \mathrm{~L}$ & 2 & recruiting & NCT02354586 \\
Niraparib + Bevacizumab & Platinum-sensitive & $>1 \mathrm{~L}$ & 2 & recruiting & NCT02354131 \\
$\begin{array}{l}\text { Niraparib + Bevacizumab } \\
\text { Niraparib + }\end{array}$ & Platinum-sensitive & 1L maintenance & 2 & recruiting & NCT03326193 \\
Pembrolizumab & Platinum-resistant & $>2 \mathrm{~L}$ & $1-2$ & recruiting & NCT02657889 \\
Niraparib + Everolimus & $\begin{array}{c}\text { Platinum-sensitive } \\
\text { and resistant }\end{array}$ & $>1 \mathrm{~L}$ & 1 & $\begin{array}{l}\text { not yet } \\
\text { recruiting }\end{array}$ & NCT03154281 \\
\hline
\end{tabular}


Table 3 Ongoing clinical trials involving Rucaparib in EOC.

\begin{tabular}{|c|c|c|c|c|c|}
\hline Combination & Setting & $\begin{array}{l}\text { Line of } \\
\text { therapy }\end{array}$ & Phase & Status & $\begin{array}{l}\text { Trial } \\
\text { identifier }\end{array}$ \\
\hline Rucaparib & Platinum sensitive & $>1$ & 2 & $\begin{array}{l}\text { Ongoing not } \\
\text { recruiting }\end{array}$ & NCT01891344 \\
\hline $\begin{array}{l}\text { Rucaparib + } \\
\text { Atetolizumab }\end{array}$ & $\begin{array}{l}\text { Gynaecological } \\
\text { neoplasms/Breast } \\
\text { cancer }\end{array}$ & $>1$ & 1 & Recruiting & NCT03101280 \\
\hline $\begin{array}{l}\text { Rucaparib vs } \\
\text { chemotherapy }\end{array}$ & $\begin{array}{l}\text { Platinum } \\
\text { sensitive/resistant }\end{array}$ & $\geq 3$ & 3 & Recruiting & NCT02855944 \\
\hline Rucaparib & $\begin{array}{l}\text { Ovarian cancer/other } \\
\text { BRCA mutated } \\
\text { tumors }\end{array}$ & $\leq 4$ & $1 / 2$ & $\begin{array}{l}\text { Ongoing not } \\
\text { recruiting }\end{array}$ & NCT01482715 \\
\hline
\end{tabular}


Table 4. Ongoing clinical trials involving Talazoparib in EOC.

\begin{tabular}{llllll}
\hline Combination & Setting & $\begin{array}{l}\text { Line of } \\
\text { therapy }\end{array}$ & Phase & Status & Trial identifier \\
\hline Talazoparib & Neoadjuvant & 1 & 1 & $\begin{array}{l}\text { Ongoing not } \\
\text { recruiting }\end{array}$ & NCT02316834 \\
$\begin{array}{l}\text { Talazoparib } \\
+ \text { avelumab }\end{array}$ & $\begin{array}{l}\text { Platinum sensitive } \\
\text { EOC/other tumors }\end{array}$ & $\geq 1$ & $1 / 2$ & Recruiting & NCT03330405 \\
Talazoparib & $\begin{array}{l}\text { Platinum sensitive } \\
\text { or platinum naive }\end{array}$ & $\geq 1$ & 1 & Recruiting & NCT01989546 \\
EOC/other tumors & $\begin{array}{l}\text { EOazoparib } \\
\text { Progression after } \\
\text { another PARPis }\end{array}$ & $\geq 1$ & 2 & Completed & \\
\hline Talazoparib & $\begin{array}{l}\text { EOC/other tumors } \\
\text { EOny }\end{array}$ & 2 & $\begin{array}{l}\text { Ongoing not } \\
\text { recruiting }\end{array}$ & NCT02286687 \\
\hline
\end{tabular}




\begin{tabular}{|c|c|c|c|c|c|}
\hline Combination & Setting & $\begin{array}{l}\text { Line of } \\
\text { therapy }\end{array}$ & Phase & Status & $\begin{array}{l}\text { Trial } \\
\text { identifier }\end{array}$ \\
\hline $\begin{array}{l}\text { Veliparib + } \\
\text { topotecan }\end{array}$ & $\begin{array}{l}\text { Platinum } \\
\text { resistant/partially } \\
\text { resistant EOC }\end{array}$ & $>1$ & $1 / 2$ & Completed & NCT01690598 \\
\hline $\begin{array}{l}\text { Veliparib + } \\
\text { topotecan }\end{array}$ & $\begin{array}{l}\text { Solid } \\
\text { tumors/relapsed } \\
\text { EOC }\end{array}$ & $<4$ & $1 / 2$ & Recruiting & NCT01012817 \\
\hline $\begin{array}{l}\text { Veliparib+ } \\
\text { Carboplatin } \\
\text { + Paclitaxel }\end{array}$ & $\begin{array}{l}\text { Newly } \\
\text { diagnosed EOC }\end{array}$ & 1 & 3 & $\begin{array}{l}\text { Ongoing not } \\
\text { recruiting }\end{array}$ & NCT02470585 \\
\hline Veliparib & Recurrent EOC & $>1$ & 2 & $\begin{array}{l}\text { Ongoing not } \\
\text { ecruiting }\end{array}$ & NCT01540565 \\
\hline $\begin{array}{l}\text { Veliparib + } \\
\text { Fluxuridine }\end{array}$ & & $>5$ & 1 & Recruiting & NCT01749397 \\
\hline $\begin{array}{l}\text { Veliparib + } \\
\text { PLD }\end{array}$ & $\begin{array}{l}\text { Recurent } \\
\text { ovarian/breast } \\
\text { cancer }\end{array}$ & $<2 \mathrm{pl}$ based & 1 & $\begin{array}{l}\text { Ongoing not } \\
\text { recruiting }\end{array}$ & NCT01145430 \\
\hline $\begin{array}{l}\text { Veliparib+ } \\
\text { Carboplatin } \\
\text { + Paclitaxel + } \\
\text { Bevacizumab }\end{array}$ & $\begin{array}{l}\text { Newly } \\
\text { diagnosed EOC }\end{array}$ & 1 & 1 & $\begin{array}{l}\text { Ongoing not } \\
\text { recruiting }\end{array}$ & NCT00989651 \\
\hline
\end{tabular}




\begin{tabular}{|c|c|c|c|c|}
\hline Patent ID & Patent Title & Short Description & $\begin{array}{c}\text { Date of } \\
\text { registration }\end{array}$ & $\begin{array}{l}\text { Reference } \\
\text { number }\end{array}$ \\
\hline WO 2013153130 A1 & $\begin{array}{l}\text { Novel markers for detecting microsatellite } \\
\text { instability in cancer and determining synthetic } \\
\text { lethality with inhibition of the DNA base } \\
\text { excision repair pathway }\end{array}$ & $\begin{array}{l}\text { New markers to detect Mismatch Repair } \\
\text { (MMR) deficient tumors. Innovative treatment } \\
\text { for MMR-deficient tumors based on the } \\
\text { synthetic lethal interaction between MMR and } \\
\text { PARP. }\end{array}$ & 10 Apr 2013 & {$[138]$} \\
\hline WO/2016/018089A1 & $\begin{array}{l}\text { Novel biomarker for predicting sensitivity to } \\
\text { parp Inhibitor, and use thereof }\end{array}$ & $\begin{array}{l}\text { A novel biomarker to predict response to } \\
\text { PARPis }\end{array}$ & 9 Aug 2013 & {$[140]$} \\
\hline $\begin{array}{l}\text { US20150290185 } \\
\text { (EP2918292A1) }\end{array}$ & $\begin{array}{l}\text { Polymeric Compound Having Camptothecin } \\
\text { Compound And Anti-Cancer Effect Enhancer } \\
\text { Bound Thereto, And Use of Same }\end{array}$ & $\begin{array}{l}\text { A polymeric camptothecin compound }+\mathrm{a} \\
\text { PARP inhibitor }\end{array}$ & 31 Oct 2013 & [141] \\
\hline $\begin{array}{c}\text { US20160138114 } \\
(\text { WO/2014/205105A1) }\end{array}$ & $\begin{array}{l}\text { Biomarkers of Response to Inhibition of Poly- } \\
\text { ADP Ribose Polymerase (PARP) in Cancer }\end{array}$ & $\begin{array}{l}\text { Methods of identifying PARPis sensitive } \\
\text { tumors }\end{array}$ & 18 Jun 2014 & [142] \\
\hline 9388472 & $\begin{array}{l}\text { Methods and materials for assessing loss of } \\
\text { heterozygosity }\end{array}$ & $\begin{array}{l}\text { Methods and materials used in assaying } \mathrm{LOH} \\
\text { signature in tumor cells }\end{array}$ & 18 Jun 2014 & [118] \\
\hline $\begin{array}{c}\text { JP2017504623A } \\
\text { (WO/2015/108986A1) }\end{array}$ & $\begin{array}{l}\text { Use of PARP inhibitor for treating the patient } \\
\text { of a breast cancer or an ovarian cancer who } \\
\text { shows loss of heterozygosis }\end{array}$ & Use of PARPis in subject with $\mathrm{LOH}$ & 14 Jan 2015 & [143] \\
\hline $\begin{array}{c}\text { US20170100368 } \\
\text { (WO 2015184145 A1) }\end{array}$ & $\begin{array}{l}\text { Use of eribulin and poly (adp ribose) } \\
\text { polymerase (parp) inhibitors as combination } \\
\text { therapy for the treatment of cancer }\end{array}$ & $\begin{array}{l}\text { Eribulin mesylate + PARP inhibithors (e.g., } \\
\text { E7449) +/- platinum based therapy }\end{array}$ & 28 May 2015 & [144] \\
\hline US20150344968 & $\begin{array}{l}\text { Methods for determining PARP inhibitor and } \\
\text { platinum resistance in cancer therapy }\end{array}$ & BRCA1 exon excision variant sequencing & 01 Jun 2015 & [145] \\
\hline WO 2016138574 A1 & Homologous recombination factors & $\begin{array}{l}\text { Factors that act on homologous recombination, } \\
\text { method to modulate it. }\end{array}$ & 1 Mar 2016 & [116] \\
\hline WO 2016209935 A1 & $\begin{array}{l}\text { Platinum compounds, compositions, and uses } \\
\text { thereof }\end{array}$ & $\begin{array}{l}\text { Platinum compounds having at least one } \\
\text { reacting group (e.g. that recognize a selected } \\
\text { target cell population) }\end{array}$ & 22 Jun 2016 & [146] \\
\hline $\begin{array}{c}9707302 \\
(\text { US20170274093) }\end{array}$ & $\begin{array}{l}\text { Combining anti-HLA-DR or anti-Trop-2 } \\
\text { antibodies with microtubule inhibitors, PARP } \\
\text { inhibitors, bruton kinase inhibitors or } \\
\text { phosphoinositide 3-kinase inhibitors } \\
\text { significantly improves therapeutic outcome in }\end{array}$ & $\begin{array}{l}\text { PARP inhibitors + adoptive cell therapy, } \\
\text { bruton kinase inhibitors or PI } 3 \mathrm{~K} \text { inhibitors }\end{array}$ & 23 Jun 2016 & [147] \\
\hline
\end{tabular}


cancer

\begin{tabular}{|c|c|c|c|c|}
\hline & cancer & & & \\
\hline WO 2017008912 A1 & $\begin{array}{l}\text { Method for determining a mutation in genomic } \\
\text { dna, use of the method and kit for carrying out } \\
\text { said method }\end{array}$ & $\begin{array}{l}\text { A mutation analysis is carried out using } \\
\text { genomic DNA }\end{array}$ & 15 Jul 2016 & [117] \\
\hline WO/2017/013237A1 & $\begin{array}{l}\text { Use of a combination of dbait molecule and } \\
\text { PARP inhibitors to treat cancer }\end{array}$ & PARP inhibitor + nucleic acid molecule & 22 Jul 2016 & [148] \\
\hline WO/2017/031442A1 & $\begin{array}{l}\text { Combination therapy using liposomal } \\
\text { irinotecan and a PARP inhibitor for cancer } \\
\text { treatment }\end{array}$ & $\begin{array}{l}\text { Irinotecan liposomal formulation (MM-398) } \\
\text { and Veliparib }\end{array}$ & 19 Aug 2016 & [132] \\
\hline WO/2017/029517A1 & $\begin{array}{l}\text { Compositions comprising phosphoinositide } 3 \text { - } \\
\text { kinase inhibitors and a second antiproliferative } \\
\text { agent }\end{array}$ & $\begin{array}{l}\text { A composition of phosphoinositide 3-kinase } \\
\text { inhibitors and a second agent (e.g. a PARPi) }\end{array}$ & 19 Aug 2016 & [149] \\
\hline US20170035737 & $\begin{array}{l}\text { Treatment of BRCA1-defective cancer or } \\
\text { resistant cancers }\end{array}$ & $\begin{array}{l}\mathrm{COH} 29((\mathrm{~N}-(4-(3,4-d i h y d r o x y p h e n y l)-5- \\
\text { phenylthiazol-2-yl)-3,4-dihydroxybenzamide }))\end{array}$ & 9 Feb 2017 & {$[125]$} \\
\hline US20170049767 & Combination therapy for cancer treatment & Topoisomerase- 1 inhibitor + PARPis & 23 Feb 2017 & [130] \\
\hline WO/2017/151554A1 & $\begin{array}{l}\text { Combination therapy for treatment of ovarian } \\
\text { cancer }\end{array}$ & $\begin{array}{l}\text { Ganetespib + a DNA- damaging or repair- } \\
\text { inhibiting agent }\end{array}$ & 28 Feb 2017 & [131] \\
\hline
\end{tabular}

